\title{
DIFFERENTIAL FORMS ON GENERAL COMMUTATIVE ALGEBRAS
}

BY

\author{
GEORGE S. RINEHART(1)
}

Introduction. Let $K$ be a commutative ring with unit, and let $R$ be a commutative unitary $K$-algebra. We shall be concerned with variously defined cohomology theories based on algebras of differential forms, where $R$ plays the role of a ring of functions.

Let $T_{R}$ be the Lie algebra of the $K$-derivations of $R$, and let $E\left(T_{R}\right)$ be the exterior algebra over $R$ of $T_{R}$. We can form $\operatorname{Hom}_{R}\left(E\left(T_{R}\right), R\right)$ and define on it the usual formal differentiation. If $R$ is the ring of functions on a $C^{\infty}$-manifold then the elements of $T_{R}$ are the differentiable tangent vector fields, and the complex $\operatorname{Hom}_{R}\left(E\left(T_{R}\right), R\right)$ is naturally isomorphic to the usual de Rham complex of differential forms. In $[5, \S \S 6-9]$ the complex $\operatorname{Hom}_{R}\left(E\left(T_{R}\right), R\right)$ is studied. It is shown that if $K$ is a field contained in $R$, and if either $R$ is an integral domain finitely ring-generated over $K$ and $T_{R}$ is $R$-projective, or $R$ is a field, then the homology of this complex may be identified with $\operatorname{Ext}_{V}(R, R)$, for a suitably defined ring $V$. $\S \S 1-6$ of the present work are primarily a straightforward generalization of the results of this portion of [5] to the case in which $K$ and $R$ are arbitrary (commutative) rings.

In making this generalization we are led naturally to replace $T_{R}$ by an arbitrary Lie algebra with an $R$-module structure which is represented as derivations of $R$ and which satisfies certain additional properties satisfied by $T_{R}$. We give these properties in $\S 2$. $L$ is essentially a quasi-Lie algebra as defined in [3]. The precise definition given corresponds to that of a $d$-Lie ring given in [8], where also the cohomology based on $\operatorname{Hom}_{R}(E(L), A)$ is defined.

In $\$ 2$ we define an associative algebra $V$ of universal differential operators generated by $R$ and $L$. In case $L$ operates trivially on $R, V$ is the usual universal enveloping algebra of the $R$-Lie algebra $L$. In $\S 3$ we prove a Poincaré-BirkhoffWitt theorem for $V$. In $\S 4$ we show that if $L$ is $R$-projective then for any $V$-module $A$ we may identify the cohomology based on $\operatorname{Hom}_{R}(E(L), A)$ with $\operatorname{Ext}_{V}(R, A)$, which we denote by $H_{R}(L, A)$. In particular, the de Rham cohomology of a $C^{\infty}$ manifold is thus identified with an $\operatorname{Ext}_{V}(R, R)$.

Received by the editors July 20, 1962.

(1) The research for this paper was carried out while the author was a National Science Foundation Fellow. The author is now at Cornell University. 
$\S 5$ deals with certain functorial properties of $H_{R}(L, A)$, and $\S 6$ with the operations which generalize the usual Lie derivation and contraction operations on the algebra of differential forms.

Let $S=R \otimes_{K} R$. There are standard products under which $\operatorname{Tor}^{S}(R, R)$ and $\operatorname{Ext}_{S}(R, R)$ become skew-commutative $R$-algebras. Let $D_{R}$ be the $R$-module of the formal differentials of $R$ (see $\S 9$ ). Let $E\left(D_{R}\right)$ be the exterior $R$-algebra built over $D_{R}$. There is an isomorphism from $D_{R}$ onto $\operatorname{Tor}_{1}^{S}(R, R)$, which, extends canonically to an algebra homomorphism from $E\left(D_{R}\right)$ into $\operatorname{Tor}^{S}(R, R)$. There is also a natural homomorphism from $\operatorname{Tor}^{s}(R, R)$ into $\operatorname{Hom}_{R}\left(\operatorname{Ext}_{S}(R, R), R\right)$. $\operatorname{Ext}_{S}^{1}(R, R)$ is isomorphic with $T_{R}$, so that $\operatorname{Ext}_{S}(R, R)$ contains a canonical homomorphic image of $E\left(T_{R}\right)$ (assuming that 2 has an inverse in $R$ ). Thus there is a homomorphism from $\operatorname{Tor}^{S}(R, R)$ into $\operatorname{Hom}_{R}\left(E\left(T_{R}\right), R\right)$.

It is shown in [5] that, if $K$ is a perfect field and $R$ is a regular affine $K$-algebra, all the homomorphisms of the preceding paragraph are isomorphisms. Hence in this case $\operatorname{Tor}^{S}(R, R)$ is an algebra of differential forms. In $\$ \S 7-10$ we are concerned with operations on $\operatorname{Tor}^{S}(R, R)$ analogous to the usual operations on differential forms, in the general case in which $K$ is an arbitrary commutative ring with unit and $R$ is a commutative unitary $K$-projective $K$-algebra. In $\S 7$ we define, in a general setting, a pairing between Ext and Tor which, in the present case, defines a right $\operatorname{Ext}_{S}(R, R)$-module structure on $\operatorname{Tor}^{S}(R, R)$. By means of this module structure, elements of $\operatorname{Ext}_{S}^{n}(R, R)$ act as endomorphisms of degree $-n$, and those endomorphisms corresponding to elements of degree 1 are anti-derivations analogous to the contraction operators on differential forms. In $\$ 9$ we define the operations on $\operatorname{Tor}^{S}(R, R)$ analogous to the usual Lie derivations of differential forms, and show that the usual relations involving contraction and Lie derivations obtain. In $\$ 10$ we define a formal differentiation map for $\operatorname{Tor}^{S}(R, R)$ generalizing the differentiation of formal differentials.

This work was done as a doctoral thesis under the direction of Professor Gerhard Hochschild, for whose encouragement and generous advice and instruction the author is deeply grateful.

1. Preliminaries. Henceforth, we shall always assume that all rings have an identity, and that all modules and ring homomorphisms are unitary.

LEMMA 1.1. Let $R$ be any ring. Let $X_{i}$, for each $i$ in some index set, be a right $R$-module, and let $A$ be a projective left $R$-module. Let " $\prod$ " denote the (strong) direct product. Then the natural homomorphism: $\left(\prod_{i} X_{i}\right) \otimes_{R} A \rightarrow$ $\prod_{i}\left(X_{i} \otimes_{R} A\right)$, is a monomorphism.

Proof. Choose a free $R$-module $F=\sum_{j} R_{j}$, where each $R_{j}$ is a copy of $R$, such that $A$ is a direct summand of $F .\left(\prod_{i} X_{i}\right) \otimes_{R} F=\sum_{j}\left(\prod_{i} X_{i}\right) \otimes_{R} R_{j}=\sum_{j} \prod_{i} X_{i j}$, where each $X_{i j}$ is a copy of $X_{i}$. The latter may be viewed as a subset of $\prod_{i} \Sigma_{j} X_{i j}=\prod_{i} \Sigma_{j}\left(X_{i} \otimes_{R} R_{j}\right)=\prod_{i}\left(X_{i} \otimes_{R} F\right)$. The lemma follows, since $\left(\prod X_{i}\right) \otimes_{R} A$ is a direct summand of $\left(\prod X_{i}\right) \otimes_{R} F$. 
If $R$ is a commutative ring and $P \subset R$ is a prime ideal $\neq R$, denote by $R_{P}$ the corresponding local ring; that is, $R_{P}$ is the set of equivalence classes of pairs $r / s$ where $r$ and $s$ are elements of $R, s \notin P$, and where $r_{1} / s_{1}$ is equivalent to $r_{2} / s_{2}$ in case there is a $v \notin P$ such that $v\left(r_{1} s_{2}-r_{2} s_{1}\right)=0$, with addition and multiplication defined in the obvious way. There is a canonical homomorphism $\beta_{P}: R \rightarrow R_{P}$ which sends $r$ onto the class of $r / 1$. The kernel of $\beta_{P}$ is $H_{P}=\{r \in R: \exists v \notin P \ni r v=0\}$. Let $\mathscr{M}$ be the set of all maximal ideals of $R$. We can define a homomorphism $\beta$ mapping $R$ into $\prod_{P \in \mathcal{M}} R_{P}$ such that the $R_{P}$-component of $\beta(r)$ is $\beta_{P}(r)$.

LEMMA 1.2. $\beta$ is a monomorphism.

Proof. Let $0 \neq r \in R$. Set $I_{r}=\{s \in R: r s=0\}$. Then $I_{r}$ is a proper ideal of $R$. Choose $P \in \mathscr{M}$ such that $P \supset I_{r}$. Then $r \notin H_{P}$. We conclude from this that $\bigcap_{P \in \mathscr{M}} H_{P}=(0)$. This proves Lemma 1.2.

2. ( $K, R)$-Lie algebras and their enveloping algebras. Let $K$ be a commutative ring, and let $R$ be a commutative $K$-algebra. In the sequel, all $R$-modules will be regarded as $K$-modules in the natural fashion $(k \cdot m=(k \cdot 1) \cdot m)$. Let $L$ be a Lie ring that is also an $R$-module. Suppose that we are given a Lie ring and $R$-module homomorphism from $L$ to the $K$-derivations of $R$. If $\mu \in L$, we will denote the image of $\mu$ under this homomorphism by $r \rightarrow \mu(r)$. Suppose finally that, for all $\alpha, \mu \in L$, and $r \in R$,

$$
[\alpha, r \mu]=r[\alpha, \mu]+\alpha(r) \mu .
$$

We will call such an $L$ a $(K, R)-L i e$ algebra.

We can make the direct $R$-module sum $R+L$ into a $K$-Lie algebra by defining

$$
[r+\alpha, s+\mu]=(\alpha(s)-\mu(r))+[\alpha, \mu] .
$$

Form the tensor algebra over $K$ of $R+L$, and factor by the usual ideal to obtain $U$, the universal enveloping algebra of the $K$-Lie algebra $R+L$. Let $U+$ be the subalgebra generated by the canonical image of $R+L$ in $U$. For $r \in R$ and $z$ an element of the $R$-module $R+L$, let $r \cdot z$ be the result of operating on $z$ with $r$, and denote by $z^{\prime}$ the canonical image of $z$ in $U^{+}$. Let $P$ be the two-sided ideal of $U^{+}$generated by all elements of the form $(r \cdot z)^{\prime}-r^{\prime} z^{\prime}$, with $r \in R$ and $z \in Z$. Define

$$
V(R, L)=U^{+} / P \text {. }
$$

A module for $K$-Lie algebra $R+L$ is called an $R$-regular $L$-module in case for all $r \in R, z \in R+L$, and $m \in M$,

$$
r \cdot(z \cdot m)=(r \cdot z) \cdot m \text {. }
$$

The canonical map: $R+L \rightarrow V(R, L)$ endows any $V(R, L)$-module with the structure of an $R$-regular $L$-module. Thus we have a one-to-one correspondence 
between $V(R, L)$-modules and $R$-regular $L$-modules. In particular, $R$ has a natural structure as an $R$-regular $L$-module, and the representation of $R$ thus obtained is faithful. Hence the map: $R \rightarrow V(R, L)$ is a monomorphism. Henceforth we will identify $R$ with its image in $V(R, L)$.

Note that if we take $K=R$, and let each element of $L$ act as the zero derivation of $K$, then $L$ is a $(K, R)$-Lie algebra, and $V(K, L)$ is the usual universal enveloping algebra of the $K$-Lie algebra $L$.

If $A$ and $B$ are $V(R, L)$-modules, we can define an $R$-regular $L$-module structure on $\operatorname{Hom}_{R}(A, B)$ such that, for $r \in R, \mu \in L, a \in A$, and $f \in \operatorname{Hom}_{R}(A, B)$, $(r \cdot h)(a)=r \cdot h(a)=h(r \cdot a)$, and $(\mu \cdot h)(a)=\mu \cdot h(a)-h(\mu \cdot a)$. We can also define an $R$-regular $L$-module structure on $A \otimes_{R} B$ such that, for $r \in R, \mu \in L$, $a \in A$, and $b \in B, r \cdot(a \otimes b)=(r \cdot a) \otimes b=a \otimes(r \cdot b)$, and $\mu \cdot(a \otimes b)=(\mu \cdot a) \otimes b$ $+a \otimes(\mu \cdot b)$. We have

Lemma 2.1. Let $B$ be a left $V(R, L)$-module. The $V(R, L)$-modules $\operatorname{Hom}_{R}(V(R, L), B)$ and $B \otimes_{R} V(R, L)$ as defined above are isomorphic respectively to $\operatorname{Hom}_{R}(V(R, L), B)$ and $V(R, L) \otimes_{R} B$ with the usual left $V(R, L)$-module structures.

The proof can be read verbatim as the proofs of Lemmas 6.1 and 6.2 of [5], substituting $L$ for $T_{R}$ and $V(R, L)$ for $V_{R}$.

3. A Poincaré-Birkhoff-Witt theorem for $V(R, L)$. Denote by $\bar{L}$ the image of $L$ in $V(R, L)$, and by $\bar{\mu}$ the image in $\bar{L}$ of $\mu \in L$. Let $V_{p}(R, L)$ be the left $R$-submodule of $V(R, L)$ generated by products of at most $p$ elements of $\bar{L}$. We have thus a filtration of $V(R, L)$. Denote by $G(V(R, L))$ the associated graded $R$ module; i.e., the direct sum of the $R$-modules $V_{p}(R, L) / V_{p-1}(R, L)$, where $V_{-1}(R, L)=(0)$. Remark that if $z \in V_{p}(R, L)$ and $r \in R, r z-z r \in V_{p-1}(R, L)$. Hence the left and right $R$-module structures on $G(V(R, L))$ are the same, and we may regard $G(V(R, L))$ as an $R$-algebra. Denote by $S(L)$ the symmetric $R$ algebra on $L$.

THEOREM 3.1. If $L$ is $R$-projective, then the canonical R-epimorphism, $S(L) \rightarrow G(V(R, L))$, is an $R$-algebra isomorphism.

Proof. First we prove the result under the assumption that $L$ is $R$-free. In doing so, we adapt the notation and proof of [1, Lemma 3.5, p. 271]. Let $\left\{\mu_{i}\right\}$ be an ordered $R$-basis of $L$. Let $u_{i}$ denote $\mu_{i}$ considered as an element of $S(L)$. If $I$ is a sequence $i_{1} \leqq \cdots \leqq i_{n}$, let $u_{I}=u_{i_{1}} \cdots u_{i_{n}}$. If $I$ is the empty sequence, let $u_{I}=1$. Write $j \leqq I$ in case either $j \leqq i_{1}$ or $I$ is empty. We will define the structure of an $R$-regular $L$-module on $S(L)$ such that, whenever $j \leqq I, \mu_{j} \cdot u_{I}=u_{j} u_{I} \cdot$ The resulting $V(R, L)$-module structure for $S(L)$ will have the property that, for any ordered sequence $\left\{i_{1}, \cdots, i_{n}\right\}=I,\left(\bar{\mu}_{i_{1}} \cdots \bar{\mu}_{i_{n}}\right) \cdot 1=u_{I}$. Noting that the $u_{I}$ 's form an $R$-basis for $S(L)$, we see that this suffices to prove Theorem 3.1. 
Let $S^{p}(L)$ denote the homogeneous component of degree $p$ of $S(L)$. Let $Q_{p}=\sum_{q=0}^{p} S^{q}(L)$. We proceed inductively to define a $K$-bilinear map from $L \times S(L)$ to $S(L)$, denoted by $(\mu, u) \rightarrow \mu \cdot u$, by defining its restriction: $L \times Q_{p} \rightarrow Q_{p+1}$ for each $p$, subject to the following conditions:

$$
\begin{aligned}
& \mu_{j} \cdot u_{I}=u_{j} u_{I} \text { whenever } j \leqq I, u_{I} \in Q_{p} ; \\
& \mu \cdot(\alpha \cdot u)=\alpha \cdot(\mu \cdot u)+[\mu, \alpha] \cdot u \text { if } \mu, \alpha \in L, u \in Q_{p-1} ; \\
& \mu_{j} \cdot u_{I}-u_{j} u_{I} \in Q_{q} \text { if } u_{I} \in Q_{q}, q \leqq p ; \\
&(r \mu) \cdot(s u)=r(s(\mu \cdot u)+\mu(s) u) \text { if } r, s \in R, \mu \in L, u \in Q_{p} .
\end{aligned}
$$

For $p=0$, define $\mu \cdot r=r \mu+\mu(r)$, satisfying (3.1) through (3.4).

Now suppose we have already defined an action: $L \times Q_{p-1} \rightarrow Q_{p}$ satisfying the conditions corresponding to (3.1) through (3.4). In order to extend this, we first define the action by the lelements $\mu_{i}$ mapping $S^{p}(L)$ into $Q_{p+1}$. We may assume inductively that we have defined this action for all $\mu_{j}$ such that $j<i$. Consider an element $u_{I} \in S^{p}(L)$. If $i \leqq I$, define $\mu_{i} \cdot u_{I}=u_{i} u_{I}$. If not, then $I=(j, J)$, with $j<i$, and we define $\mu_{i} u_{I}=\mu_{j} \cdot\left(\mu_{i} \cdot u_{J}\right)+\left[\mu_{i}, \mu_{j}\right] \cdot u_{J}$. Now we define the action by $\mu_{i}$ on all of $S^{p}(L)$ by defining $\mu_{i}\left(r u_{I}\right)=r\left(\mu_{i} \cdot u_{I}\right)+\mu_{i}(r) u_{I}$ if $r \in R$, and extending by $K$-linearity. Thus we have defined the action by the elements $\mu_{i}$. To define the action on $S^{p}(L)$ by an arbitrary element of $L$, define $\left(r \mu_{i}\right) \cdot u$ $=r\left(\mu_{i} \cdot u\right)$ if $r \in R, u \in S^{p}(L)$, and extend by $K$-linearity. Conditions (3.1), . (3.3), and (3.4) are clearly satisfied. The verification that

$$
\mu_{j} \cdot\left(\mu_{k} \cdot u_{I}\right)=\mu_{k} \cdot\left(\mu_{j} \cdot u_{I}\right)+\left[\mu_{j}, \mu_{k}\right] \cdot u_{I} \quad \text { if } u_{I} \in S^{p-1}(L)
$$

does not involve consideration of the $R$-module structure and so is identical with the corresponding part of the proof that we are adapting $[1$, p. 273]. Using this and (3.4), together with the property of $L$ assumed earlier, (Equation (2.1)) the verification of (3.2) is a straightforward computation. Thus we have an action by elements of $L$ on $S(L)$. We use this to define an action of $R+L$ on $S(L)$ in the obvious way. Using (3.2) and (3.4) one sees easily that this endows $S(L)$ with the structure of an $R$-regular $L$-module. This proves Theorem 2.1 when $L$ is $R$-free.

Now assume only that $L$ is $R$-projective. Let $P$ be any prime ideal of $R$. Consider the $K$-algebra $R_{P}$. If $\mu$ is any $K$-derivation of $R$, the formula $\mu(r / s)=(s \mu(r)-r \mu(s)) / s^{2}$ extends $\mu$ to a $K$-derivation of $R_{P}$. Thus $L$ is represented on $R_{P}$. Let $L_{P}=R_{P} \otimes_{R} L$ with the natural $R_{P}$-module structure. We can define a commutation on $L_{P}$ such that, for $x, y \in R_{P}, \alpha, \mu \in L$,

$$
[x \otimes \alpha, y \otimes \mu]=x y \otimes[\alpha, \mu]-y \mu(x) \otimes \alpha+x \alpha(y) \otimes \mu .
$$

(This commutator is clearly additive in all four terms. Hence it is only necessary to verify that, e.g., $[r x \otimes \alpha, y \otimes \mu]=[x \otimes r \alpha, y \otimes \mu]$ for $r \in R$. Using (2.1), this 
is a straightforward computation.) The commutation is clearly anti-commutative, and one checks without difficulty that it satisfies the Jacobi identity. Thus $L_{p}$ becomes a Lie algebra. The elements of $L_{P}$ act as derivations of $R_{P}$ in the natural fashion, and it is immediate that this gives a representation of the $K$-Lie algebra $L_{P}$, and that $L_{P}$ thus becomes a $\left(K, R_{P}\right)$-Lie algebra. Let $V(P)=V\left(R_{P}, L_{P}\right)$.

Since $L$ is $R$-projective, so is $S(L)$, and hence the monomorphism $\beta$ of Lemma 1.2 induces a monomorphism: $S(L)=R \otimes_{R} S(L) \rightarrow\left(\prod R_{P}\right) \otimes_{R} S(L)$, where the product is taken over all maximal ideals of $R$. By Lemma 1.1, the latter is naturally injected into $\prod\left(R_{P} \otimes S(L)\right)=\prod S\left(L_{P}\right)$. The natural $R$-module and Lie algebra homomorphism: $R+L \rightarrow R_{P}+L_{P}$ defines an $R$-algebra homomorphism: $V(R, L) \rightarrow V(P)$. These in turn yield a map: $V(R, L) \rightarrow \prod V(P)$. This map is compatible with the filtration of $V(R, L)$ and the $V(P)$ 's, so that we obtain a map: $G(V(R, L)) \rightarrow \prod G(V(P))$. Since $L$ is $R$-projective, $L_{P}$ is $R_{P}$-projective. Hence, since $R_{P}$ is a local ring, $L_{P}$ is $R_{P}$-free [7]. By the first part of the proof of this lemma, the map $S\left(L_{P}\right) \rightarrow G(V(P))$ is therefore an isomorphism. Hence we have the commutative and exact diagram

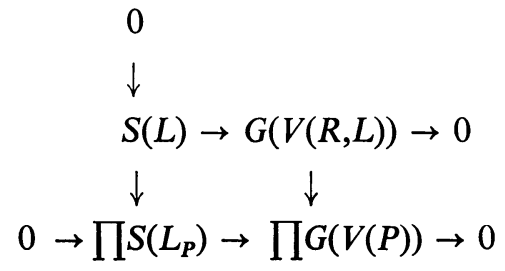

from which we deduce that the top row is a monomorphism. This completes the proof of Theorem 3.1.

Note that in the particular case in which $R=K$ and $L$ operates trivially, this theorem is the statement that the usual Poincaré-Birkhoff-Witt theorem holds whenever $L$ is $K$-projective.

4. $\operatorname{Ext}_{V}(R, A)$ as a cohomology of differential forms. Let $E(L)$ denote the graded exterior $R$-algebra over $L$. Consider the graded group $V(R, L) \otimes_{R} E(L)$ where $V(R, L)$ is an $R$-module by right multiplication by elements of $R$, and where the graded components are $V(R, L) \otimes_{R} E^{n}(L)$. We wish to show the existence of an endomorphism $d$ of degree -1 on this group such that, for $v \in V(R, L)$ and $\mu_{i} \in L$,

$$
\begin{aligned}
d\left(v \otimes \mu_{1} \cdots \mu_{n}\right)= & \sum_{i=1}^{n}(-1)^{i-1} v \bar{\mu}_{i} \otimes \mu_{1} \cdots \hat{\mu}_{i} \cdots \mu_{n} \\
& +\sum_{j<k}(-1)^{+k} v \otimes\left[\mu_{j}, \mu_{k}\right] \mu_{1} \cdots \hat{\mu}_{j} \cdots \hat{\mu}_{k} \cdots \mu_{n}
\end{aligned}
$$

where " " " indicates that the corresponding term is omitted. In order to do so, we define a map $\bar{d}$ from the cartesian product of $V(R, L)$ with $n$ copies of $L$ to 
$V(R, L) \otimes_{R} E^{n-1}(L)$ such that $\bar{d}\left(v, \mu_{1}, \cdots, \mu_{n}\right)$ is the right-hand side of (4.1). Then $d$ is clearly additive in each component. In $V(R, L)$

$$
r \bar{\mu}=\bar{\mu} r-\mu(r) \text { for } r \in R, \mu \in L .
$$

Using this and (2.1), it is a somewhat long but straightforward computation that, for $r \in R$,

$$
\bar{d}\left(v, r \mu_{1}, \mu_{2}, \cdots, \mu_{n}\right)=\bar{d}\left(v, \mu_{1}, \cdots, r \mu_{i}, \cdots, \mu_{n}\right)
$$

for every $i$. Also, one checks without difficulty that $\vec{d}\left(v, \mu_{1}, \cdots, \mu_{n}\right)=0$ whenever $\mu_{i}=\mu_{j}$ with some $i \neq j$. Hence $d$ induces a map: $V(R, L) \times E^{n}(L) \rightarrow$ $V(R, L) \otimes_{R} E^{n-1}(L)$. Finally, again using (2.1) and (4.2), one verifies that, for $r \in R$,

$$
\bar{d}\left(v r, \mu_{1}, \cdots, \mu_{n}\right)=\bar{d}\left(v, r \mu_{1}, \mu_{2}, \cdots, \mu_{n}\right) .
$$

Hence $\bar{d}$ induces the desired map $d$.

Define $d: V(R, L) \otimes_{R} E^{0}(L)=V(R, L) \rightarrow R$ by $d(v)=v \cdot 1$. Let $X$ denote the resulting augmented graded group. One checks directly that $d^{2}=0$ on $X$. If we give $X$ the usual $V(R, L)$-module structure $\left(v_{1} \cdot\left(v_{2} \otimes \mu_{1} \cdots \mu_{n}\right)=v_{1} v_{2} \otimes \mu_{1} \cdots \mu_{n}\right)$ then $d$ is visibly $V(R, L)$-linear.

Now assume that $L$ is $R$-projective. Then so is $E(L)$, and hence each $V(R, L) \otimes_{R} E^{n}(L)$ is $V(R, L)$-projective. Therefore $(X, d)$ is a $V(R, L)$-projective complex over $R$. We wish to show that this is actually a projective resolution; i.e., that $X$ is acyclic. In fact, we do more: We show that $X$ has an $R$-homotopy and so also defines a $(V(R, L), R)$-projective resloution of $R$, in the sense of [4].

Define $X_{p}=R+\sum_{q} V_{p-q}(R, L) \otimes E^{q}(L)$ for $p \geqq 0$, and $X_{p}=(0)$ otherwise. Note that since each $E^{q}(L)$ is $R$-projective, $X_{p}$ may be identified with its canonical image in $X$. Each $X_{p}$ is visibly stable under $d$, and we have thus defined a filtration of $X$ by $R$-subcomplexes. The associated graded complex can be identified with $G(V(R, L)) \otimes_{R} E(L)$ (augmented over $R$ ). Denote this by

$$
G(X)=\sum_{p} G^{p}(X)=\sum_{p} X_{p} / X_{p-1}
$$

By Theorem 3.1, the latter is $R$-isomorphic with $S(L) \otimes_{R} E(L)$ (augmented over $R)$. The boundary map induced by $d$ on the latter is given by

$$
d\left(u \otimes \mu_{1} \cdots \mu_{n}\right)=\sum_{i=1}^{n}(-1)^{i-1} u \mu_{i} \otimes \mu_{1} \cdots \hat{\mu}_{i} \cdots \mu_{n} .
$$

Let $L$ be a direct summand of a free $R$-module $F$. Then $S(L) \otimes_{R} E(L)$ becomes a direct $R$-complex summand of the usual Koszul complex $S(F) \otimes_{R} E(F)$. This has an $R$-homotopy [4, p. 259], which induces an $R$-homotopy on $S(L) \otimes_{R} E(L)$. Hence we have an $R$-homotopy $h$ on $G(X)$. Further, the homotopy of the Koszul complex is such that each $G^{p}(X)$ is stable under $h$.

Since, by Theorem 3.1, $V_{p}(R, L) / V_{p-1}(R, L)$ is $R$-isomorphic with $S^{p}(L), X_{p} / X_{p-1}$ is $R$-projective. Hence the sequence $0 \rightarrow X_{p-1} \rightarrow X_{p} \rightarrow X_{p} / X_{p-1} \rightarrow 0$ splits. Hence, 
by induction on $p$, there is an $R$-isomorphism $\alpha: X \rightarrow G(X)$ such that $\alpha\left(X_{p}\right)$ $=\sum_{q=0} G^{q}(X)$, and $(\alpha d-d \alpha)\left(X_{p}\right) \subset \sum_{q=0}^{p-} G^{q}(X) .\left(^{2}\right)$ Setting $g_{0}=\alpha^{-1} h \alpha$ and $g=2 g_{k-1}-g_{k-1} d g_{k-1}-d g_{k-1}$, we verify inductively that $\left(g_{k} d+d g_{k}-1\right)\left(X_{p}\right)$ $\subset X_{p-2^{k}}$, and $\left(g_{k}-g_{k-1}\right)\left(X_{p}\right) \subset X_{p-2^{k-1}}$. Hence we can define an $R$-endomorphism $g$ of $X$ to coincide with $g_{k}$ on $X_{2^{k}-1}$. Then $g d+d g=1$; i.e., $g$ is the desired homotopy.

Note that the existence of the isomorphism $\alpha$ shows incidentally that $X$ is $R$-projective.

We have proved

LEMMA 4.1. If $L$ is an $R$-projective $(K, R)$-Lie algebra, then the complex $V(R, L) \otimes_{R} E(L)=X$, as defined above, is a $V(R, L)$-projective resolution of $R$ which has an $R$-homotopy. $X$ is $R$-projective.

If $L$ is a $(K, R)$-Lie algebra, and $A$ is an $R$-regular $L$-module, we will write

$$
H_{R}(L, A)=\operatorname{Ext}_{V(R, L)}(R, A) .
$$

Note that, if $L$ is represented trivially on $K, H_{K}(L, A)$ is the usual Lie algebra cohomology of $L$.

If $L$ is $R$-projective then, by Lemma $4.1, H_{R}(L, A)$ is the homology of the complex $\operatorname{Hom}_{V(R, L)}\left(V(R, L) \otimes_{R} E(L), A\right)=\operatorname{Hom}_{R}(E(L), A)$. If we write the elements of the latter as $R$-multilinear maps with arguments in $L$ and values in $A$, which are strongly alternating in the sense that they vanish whenever two arguments are equal, the boundary map is given by

$$
\begin{aligned}
(D f)\left(\mu_{1}, \cdots, \mu_{n}\right)= & \sum_{i=1}^{n}(-1)^{i-1} \mu_{i}\left(f\left(\mu_{1}, \cdots, \hat{\mu}_{i}, \cdots, \mu_{n}\right)\right) \\
& +\sum_{j<k}(-1)^{j+k} f\left(\left[\mu_{j}, \mu_{k}\right], \mu_{1}, \cdots, \hat{\mu}_{j}, \cdots, \hat{\mu}_{k}, \cdots, \mu_{n}\right) .
\end{aligned}
$$

Hence we have

THEOREM 4.2. If $L$ is an $R$-projective $(K, R)$-Lie algebra, then $H_{R}(L, A)$ is the cohomology $K$-space based on the strongly alternating $R$-multilinear maps from $L$ to $A$ under the usual formal differentiation map.

In particular, let $M$ be a real $C^{\infty}$ manifold, and let $R$ be the ring of the differentiable real-valued functions on $M$. Let $K$ be the reals, and let $T$ be the $C^{\infty}$ vector fields on $M$. Then $T$ is a $(K, R)$-Lie algebra in the natural fashion. Moreover, $T$ is $R$-projective. Indeed, $M$ can be $C^{\infty}$-imbedded in Euclidean space of sufficiently high dimension. Then a subset of the tangent bundle over the latter forms a trivial bundle over $M$, and this bundle is the direct sum of the tangent bundle and the normal bundle over $M$. Hence $T$, the cross sections of

(2) For the details of this last section of the proof, see the last part of the proof of Theorem 7.1 in [5]. 
the tangent bundle over $M$, is a direct summand of the cross sections of a trivial bundle, and the latter form a free $R$-module. Hence Theorem 4.2 holds, and we conclude that the de Rham cohomology of $M$ is $\operatorname{Ext}_{V(R, T)}(R, R)$.

5. Functorial properties of $H_{R}(L, A) \cdot H_{R}(L, A)$ is a covariant functor of $A$, and as such associates with $V(R, L)$-exact sequences $0 \rightarrow B \rightarrow A \rightarrow C \rightarrow 0$ connecting homomorphisms $H_{R}(L, C) \rightarrow H_{R}^{n+1}(L, B)$ as usual. Moreover, $H_{R}(L, A)$ is a contravariant functor of pairs $(R, L)$ in the following sense: Let $R$ and $R^{\prime}$ be $K$-algebras, and let $L$ and $L^{\prime}$ be $(K, R)$ - and $\left(K, R^{\prime}\right)$-Lie algebras, respectively. Suppose we have a $K$-algebra homomorphism from $R$ to $R^{\prime}$ (denoted $r \rightarrow r^{\prime}$ ) and a Lie algebra homomorphism from $L$ to $L^{\prime}$ (denoted $\mu \rightarrow \mu^{\prime}$ ). Suppose further that $(r \mu)^{\prime}=r^{\prime} \mu^{\prime}$ and $(\mu(r))^{\prime}=\mu^{\prime}\left(r^{\prime}\right)$ for all $r \in R, \mu \in L$. Then we obtain a $K$-Lie algebra homomorphism from $R+L$ to $R^{\prime}+L^{\prime}$, and thus a ring homomorphism from $V(R, L)$ to $V\left(R^{\prime}, L^{\prime}\right)$, in the natural fashion. Write $V(R, L)=V, V\left(R^{\prime}, L^{\prime}\right)=V^{\prime}$. Let $X$ be a $V$-projective resolution of $R$. The homomorphism $V \rightarrow V^{\prime}$ yields a $V$-module structure for every $V^{\prime}$-module. $V^{\prime} \otimes_{V} X$ is a $V^{\prime}$-projective complex over $V^{\prime} \otimes_{V} R$, and the homomorphism $R \rightarrow R^{\prime}$ yields a natural epimorphism from the latter onto $R^{\prime}$. Thus $V^{\prime} \otimes_{V} X$ is a $V^{\prime}$-projective complex over $R^{\prime}$. Let $X^{\prime}$ be a $V^{\prime}$-projective resolution of $R^{\prime}$. Then, as usual, there is a map from $V^{\prime} \otimes_{V} X$ to $X^{\prime}$ over the identity; and there is thus induced, for each $V^{\prime}$-module $A$, a unique map from $\operatorname{Ext}_{V^{\prime}}\left(R^{\prime}, A\right)$ to the homology of $\mathrm{Hom}_{V^{\prime}}\left(V^{\prime} \otimes_{V} X, A\right)$. But the latter is canonically isomorphic to $\operatorname{Hom}_{V}(X, A)$. Hence we have a well-defined map from $H_{R^{\prime}}\left(L^{\prime}, A\right)$ to $H_{R}(L, A)$.

Now suppose that the natural map: $R^{\prime} \otimes_{R} L \rightarrow L^{\prime}$ is surjective, so that $V^{\prime}$ is generated by elements of the form $s \mu_{1}^{\prime} \cdots \mu_{n}^{\prime}$ with $s \in R^{\prime}, \mu_{1}, \cdots, \mu_{n} \in L$. Then the above map: $V^{\prime} \otimes_{V} R \rightarrow R^{\prime}$ has an inverse given by $s \rightarrow s \otimes 1$. Hence $V^{\prime} \otimes_{V} R$ and $R^{\prime}$ are isomorphic, so that $V^{\prime} \otimes_{V} X$ is a projective resolution of $R^{\prime}$ precisely when it is acyclic; i.e., when $\operatorname{Tor}_{n}^{V}\left(V^{\prime}, R\right)=(0)$ for all $n \geqq 1$. If this is the case, then the map $H_{R^{\prime}}\left(L^{\prime}, A\right) \rightarrow H_{R}(L, A)$ is an isomorphism.

In particular, suppose that $L$ is $R$-projective. We can make $R^{\prime} \otimes_{R} L$ into a $\left(K, R^{\prime}\right)$-Lie algebra in the natural fashion (cf. Equation 3.5). By Lemma 4.1, $\operatorname{Tor}^{V}\left(V\left(R^{\prime}, R^{\prime} \otimes_{R} L\right), R\right)$ is the homology of the complex $V\left(R^{\prime}, R^{\prime} \otimes_{R} L\right) \otimes_{V} V \otimes_{R} E(L)$ $=V\left(R^{\prime}, R^{\prime} \otimes_{R} L\right) \otimes_{R} E(L)=V\left(R^{\prime}, R^{\prime} \otimes_{R} L\right) \otimes_{R^{\prime}} R^{\prime} \otimes_{R} E(L)=V\left(R^{\prime}, R^{\prime} \otimes_{R} L\right)$ $\otimes_{R} E\left(R^{\prime} \otimes_{R} L\right)$. But $R^{\prime} \otimes_{R} L$ is $R^{\prime}$-projective, and hence by Lemma 4.1 the latter has zero homology in positive degrees. Hence

$$
H_{R^{\prime}}\left(R^{\prime} \otimes_{R} L, A\right) \cong H_{R}(L, A) .
$$

(Note by Theorem 4.2 this isomorphism is just that induced by the canonical identifications $\operatorname{Hom}_{R^{\prime}}\left(E\left(R^{\prime} \otimes_{R} L\right), A\right)=\operatorname{Hom}_{R^{\prime}}\left(R^{\prime} \otimes_{R} E(L), A\right)=\operatorname{Hom}_{R}(E(L), A)$.) Hence in the projective case we may always assume $R=R^{\prime}$.

Product. Let $L$ be $R$-projective. Write $V=V(R, L)$. Then $V$ is $R$-projective by Theorem 4.2. Hence if $X$ is a $V$-projective resolution of $R$, it is also $R$-projective. 
Therefore, by Lemma 2.1, $X \otimes_{R} X$ is still $V$-projective. $X \otimes_{R} X$ is a complex over $R \otimes_{R} R=R$, and its homology is $\operatorname{Tor}^{R}(R, R)$, which is zero in degree $\geqq 1$. Hence $X \otimes_{R} X$ is again a $V$-projective resolution of $R$. Therefore the natural map $\operatorname{Hom}_{V}(X, A) \otimes_{K} \operatorname{Hom}_{V}(X, B) \rightarrow \operatorname{Hom}_{V}\left(X \otimes_{R} X, A \otimes_{R} B\right)$ induces a product map $H_{R}(L, A) \otimes_{K} H_{R}(L, B) \rightarrow H_{R}\left(L, A \otimes_{R} B\right)$. One proves as usual that this product is associative, and, in case $A=B=R$, anti-commutative.

The product is induced by the usual "shuffle product" for alternating maps on the complex of Theorem 4.2. See [5, $\S 9]$ for details.

6. Operations on $H_{R}(L, A)$. Let $V$ be any ring, and let $A$ and $B$ be left $V$-modules. Let $\beta$ be a derivation of $V$. A pair of homomorphisms $f, \mu: A \rightarrow B$ will be called a $\beta$-pair in case $f$ is $V$-linear, and $\mu(v a)=v \mu(a)+\beta(v) f(a)$ for every $a \in A, v \in V$. A similar definition holds for right modules. The following is shown in [6]: Let $X$ and $Y$ be $V$-projective resolutions of $A$ and $B$, respectively. Then there is a $\beta$-pair $(\bar{f}, \bar{\mu}): X \rightarrow Y$, where $\bar{f}$ and $\bar{\mu}$ are of degree zero, commute with the boundary, and lie over $f$ and $\mu$, respectively, in the usual sense. The pair $(\bar{f}, \bar{\mu})$ is unique up to homotopy. If $C$ is a left $V$-module, and $(h, \alpha): C \rightarrow C$ is a $\beta$-pair, we define a map $\operatorname{Hom}_{V}(Y, C) \rightarrow \operatorname{Hom}_{V}(X, C)$ carrying $g \in \operatorname{Hom}_{V}(Y, C)$ onto $\alpha g \hat{f}-h g \bar{\mu}$. This induces a uniquely defined map $\operatorname{Ext}_{V, \beta}((f, \mu),(h, \alpha)): \operatorname{Ext}_{V}(B, C) \rightarrow \operatorname{Ext}_{V}(A, C)$. Similarly, if $D$ is a right $V$-module and $(h, \alpha): D \rightarrow D$ is a $\beta$-pair, we define a map $D \otimes_{V} X \rightarrow D \otimes_{V} Y$ such that, if $d \in D$ and $x \in X, d \otimes x$ is carried onto $\alpha(d) \otimes \bar{f}(x)+h(d) \otimes \bar{\mu}(x)$. This induces a uniquely defined map $\operatorname{Tor}^{V, \beta}((h, \alpha),(f, \mu)): \operatorname{Tor}^{V}(D, A) \rightarrow \operatorname{Tor}^{V}(D, B)$.

We will frequently have cause to consider pairs $(1, \alpha)$ in which the linear map is the identity. To simplify notation, we denote such pairs by $\alpha$, and write, e.g., $\operatorname{Ext}_{V, \beta}((f, \mu), \alpha)$ for $\operatorname{Ext}_{V, \beta}((f, \mu),(1, \alpha))$.

Let $T$ be a $(K, R)$-Lie algebra, and let $L$ be an ideal of $T$ which is also an $R$-submodule. Let $\mu \in T$. Then $\mu$ defines a $K$-derivation of $R+L$ by operation on $R$ and commutation on $L$, and this in turn extends to a $K$-derivation of the ring $V(R, L)$. Denote this derivation by $\beta_{\mu}$, and continue to write $V$ for $V(R, L)$. If $B$ is any $R$-regular $T$-module, let $\mu_{B}$ be the $K$-linear endomorphism of $B$ corresponding to $\mu$. Then $\left(1, \mu_{B}\right)$ is a $\beta_{\mu}$-pair. Hence we obtain an endomorphism $\operatorname{Ext}_{V, \beta_{\mu}}\left(\mu_{B}, \mu_{A}\right)$ of $\operatorname{Ext}_{V}(B, A)$ for any $V$-modules $A$ and $B$. Denote this endomorphism by $\theta_{\mu}$.

Let $\alpha \in T$. Note that the commutator $\left[\beta_{\mu}, \beta_{\alpha}\right]$ is the map $\beta_{[\mu, \alpha]}$. Hence, if $\left(1, \bar{\mu}_{B}\right)$ is a $\beta_{\mu}$-pair over $\left(1, \mu_{B}\right)$ and $\left(1, \bar{\alpha}_{B}\right)$ is a $\beta_{\alpha}$-pair over $\left(1, \alpha_{B}\right),\left(1,\left[\bar{\mu}_{B}, \bar{\alpha}_{B}\right]\right)$ is a $\beta_{[\mu, \alpha]}$-pair over $\left(1,\left[\mu_{B}, \alpha_{B}\right]\right)=\left(1,[\mu, \alpha]_{B}\right)$. Hence we conclude

$$
\left[\theta_{\mu}, \theta_{\alpha}\right]=\theta_{[\mu, \alpha]} \text {. }
$$

If $\mu \in L$ and $X$ is a $V$-projective resolution of $B$, then we can choose $\bar{\mu}_{B}=\mu_{X}$, and by $V$-linearity the resulting map on $\operatorname{Hom}_{V}(X, A)$ will be zero. Hence

PROPOSITION 6.1. If $\mu \in L, \theta_{\mu}=0$. 
Therefore $\mu \rightarrow \theta_{\mu}$ induces a representation of the $K$-Lie algebra $T / L$ on $\operatorname{Ext}_{V}(B, A)$.

Now we restrict our attention to the case in which $B=R$ and $L$ is $R$-projective. Then we have

Proposition 6.2. For every $\mu \in T, \theta_{\mu}$ is a derivation of $H_{R}(L, A)$ with respect to the product defined in the previous section.

Proof. Let $X$ be a $V$-projective resolution of $R$. Let $\left(1, \bar{\mu}_{R}\right)$ be a $\beta_{\mu}$-pair on $X$ over $\left(1, \mu_{R}\right)$. Then there is a $K$-linear map $\mu_{R}^{*}$ of $X \otimes_{R} X$ such that $\mu_{R}^{*}(x \otimes y)=\bar{\mu}_{R}(x) \otimes y+x \otimes \bar{\mu}_{R}(y)$. It is easily verified that $\left(1, \mu_{R}^{*}\right)$ is a $\beta_{\mu}$-pair over $\left(1, \mu_{R}\right)$. Denote by $\bar{\theta}_{\mu}$ the maps on $\operatorname{Hom}_{V}(X, A)$ and $\operatorname{Hom}_{V}(X, B)$ corresponding to $\bar{\mu}_{R}$, and the map on $\operatorname{Hom}_{V}\left(X \otimes_{R} X, A \otimes_{R} B\right)$ corresponding to $\mu_{R}^{*}$. Then, for $f \in \operatorname{Hom}_{V}(X, A)$ and $g \in \operatorname{Hom}_{V}(X, B)$, we have $\bar{\theta}_{\mu}(f g)=\bar{\theta}_{\mu}(f \otimes g)=\mu_{A \otimes B}(f \otimes g)$ $-(f \otimes g) \mu_{R}^{*}=\left(\mu_{A} f\right) \otimes g+f \otimes\left(\mu_{B} g\right)-(f \otimes g)\left(\bar{\mu}_{R} \otimes 1+1 \otimes \bar{\mu}_{R}\right)=\left(\mu_{A} f\right) \otimes g$ $-\left(f \bar{\mu}_{R}\right) \otimes g+f \otimes\left(\mu_{B} g\right)-f \otimes\left(g \bar{\mu}_{R}\right) !=\bar{\theta}_{\mu}(f) \otimes g+f \otimes \bar{\theta}_{\mu}(g)$. This'. proves the proposition.

Now let $X$ be the resolution of Lemma 4.1. Then it is readily seen that we may choose $\bar{\mu}_{R}$ such that

$$
\bar{\mu}_{R}\left(v \otimes \mu_{1} \cdots \mu_{n}\right)=\beta_{\mu}(v) \otimes \mu_{1} \cdots \mu_{n}+\sum_{i=1}^{n} v \otimes \mu_{1} \cdots\left[\mu, \mu_{i}\right] \cdots \mu_{n} .
$$

The resulting map on $\operatorname{Hom}_{R}(E(L), A)$ is given by $\left(\bar{\theta}_{\mu} f\right)\left(\mu_{1}, \cdots, \mu_{n}\right)=\mu\left(f\left(\mu_{1}, \cdots, \mu_{n}\right)\right)$ - $\sum_{i=1}^{n} f\left(\mu_{1}, \cdots,\left[\mu, \mu_{i}\right], \cdots, \mu_{n}\right)$, from which we see

Proposition 6.3. If $L$ is $R$-projective, then $\theta_{\mu}$ is induced by the usual Lie derivation of degree zero, corresponding to $\mu$, of the complex of differential forms.

Suppose $\mu \in L$. Then by the proof of Proposition 6.1, $\theta_{\mu}$ is homotopic to zero; i.e., there exists an endomorphism $c_{\mu}$ of degree -1 on $\operatorname{Hom}_{R}(E(L), A)$ such that

$$
c_{\mu} d+d c_{\mu}=\theta_{\mu} \text {. }
$$

We may choose $c_{\mu}$ to be the usual contraction corresponding to $\mu$,

$$
\left(c_{\mu} f\right)\left(\mu_{1}, \cdots, \mu_{n}\right)=f\left(\mu, \mu_{1}, \cdots, \mu_{n}\right) ;
$$

6.1 holds and becomes the familiar relation among these three maps on the algebra of differential forms.

7. A pairing for Ext and Tor. Let $S$ be a ring. Throughout this section we will write Ext for Ext $_{s}$ and Tor for Tor ${ }^{S}$. Consider an $S$-exact sequence

$$
0 \rightarrow E \rightarrow X_{n-1} \rightarrow \cdots \rightarrow X_{0} \rightarrow A \rightarrow 0
$$

of left $S$-modules. Denote the homomorphisms in the above sequence by $d$, and write $E=d\left(X_{n}\right)$. Then, for $0 \leqq k<n$ and $m \geqq 0$, the exact sequence 


$$
0 \rightarrow d\left(X_{k+1}\right) \rightarrow X_{k} \rightarrow d\left(X_{k}\right) \rightarrow 0
$$

yields a connecting homomorphism

$$
\operatorname{Ext}^{m}\left(d\left(X_{k+1}\right), C\right) \rightarrow \operatorname{Ext}^{m+1}\left(d\left(X_{k}\right), C\right)
$$

where $C$ is an arbitrary $S$-module. Iterating these, we obtain a homomorphism:

$$
\operatorname{Ext}^{m}(E, C) \rightarrow \operatorname{Ext}^{m+n}(A, C)
$$

Similarly we obtain homomorphisms

$$
\begin{aligned}
& \operatorname{Ext}^{m}(C, A) \rightarrow \operatorname{Ext}^{m+n}(C, E), \\
& \operatorname{Tor}_{m+n}(D, A) \rightarrow \operatorname{Tor}_{m}(D, E)
\end{aligned}
$$

where $D$ is any right $S$-module. These are called the iterated connecting homomorphisms corresponding to (7.1).

Now suppose that (7.1) has been obtained from a projective resolution, $X$, of $A$, by setting $E=d\left(X_{n}\right)$. Equation (7.2) can be imbedded in an exact sequence

$$
\operatorname{Ext}^{m}\left(X_{k}, C\right) \rightarrow \operatorname{Ext}^{m}\left(d\left(X_{k+1}\right), C\right) \rightarrow \operatorname{Ext}^{m+1}\left(d\left(X_{k}\right), C\right) \rightarrow 0
$$

and $\operatorname{Ext}^{m}\left(X_{k}, C\right)=(0)$ unless $m=0$. Hence (7.3) can be imbedded in an exact sequence

$$
\operatorname{Ext}^{m}\left(X_{n-1}, C\right) \rightarrow \operatorname{Ext}^{m}\left(d\left(X_{n}\right), C\right) \rightarrow \operatorname{Ext}^{m+n}(A, C) \rightarrow 0 .
$$

Similarly, (7.4) can be imbedded in an exact sequence

$$
0 \rightarrow \operatorname{Tor}_{m+n}(D, A) \rightarrow \operatorname{Tor}_{m}\left(D, d\left(X_{n}\right)\right) \rightarrow \operatorname{Tor}_{m}\left(D, X_{n-1}\right) \text {. }
$$

Let $n \geqq 1, m \geqq 0$. For $h \in \operatorname{Hom}_{S}\left(d\left(X_{n}\right), B\right)$, where $B$ is a left $S$-module, consider the compositions

$$
\begin{aligned}
& \operatorname{Ext}^{m}(B, C) \stackrel{h^{*}}{\rightarrow} \operatorname{Ext}^{m}\left(d\left(X_{n}\right), C\right) \rightarrow \operatorname{Ext}^{m+n}(A, C), \\
& \operatorname{Tor}_{m+n}(D, A) \rightarrow \operatorname{Tor}_{m}\left(D, d\left(X_{n}\right)\right) \stackrel{h^{*}}{\rightarrow} \operatorname{Tor}_{m}(D, B)
\end{aligned}
$$

where $h^{*}$ is the map induced by $h$.

If $h$ is the restriction to $d\left(X_{n}\right)$ of an element of $\operatorname{Hom}_{S}\left(X_{n-1}, B\right)$, then the maps (7.5) are zero. Indeed, in this case $h$ is a composition $d\left(X_{n}\right) \rightarrow X_{n-1} \rightarrow B$, so that the sequences (7.5) factor:

$$
\begin{aligned}
& \operatorname{Ext}^{m}(B, C) \rightarrow \operatorname{Ext}^{m}\left(X_{n-1}, C\right) \rightarrow \operatorname{Ext}^{m}\left(d\left(X_{n}\right), C\right) \rightarrow \operatorname{Ext}^{m+n}(A, C), \\
& \operatorname{Tor}_{m+n}(D, A) \rightarrow \operatorname{Tor}_{m}\left(D, d\left(X_{n}\right)\right) \rightarrow \operatorname{Tor}_{m}\left(D, X_{n-1}\right) \rightarrow \operatorname{Tor}_{m}(D, B) .
\end{aligned}
$$

In these latter, the last three terms of the first and the first three terms of the second form exact sequences. Hence both compositions are zero.

Then, via the exact sequence 


$$
\operatorname{Hom}_{S}\left(X_{n-1}, B\right) \rightarrow \operatorname{Hom}_{S}\left(d\left(X_{n}\right), B\right) \rightarrow \operatorname{Ext}^{n}(A, B) \rightarrow 0,
$$

the mappings which send $h$ to the sequences (7.5) define actions by elements of $\operatorname{Ext}^{n}(A, B)$ mapping $\operatorname{Ext}^{m}(B, C)$ into $\operatorname{Ext}^{m+n}(A, C)$ and $\operatorname{Tor}_{m}(D, A)$ into $\operatorname{Tor}_{m-n}(D, B)$, where we consider $\operatorname{Tor}_{k}=0$ for $k<0$. If $\beta \in \operatorname{Ext}^{n}(A, B), \mu \in \operatorname{Ext}^{m}(B, C)$, and $\alpha \in \operatorname{Tor}_{m}(D, A)$, denote these actions by $\mu \rightarrow \beta \mu$ and $\alpha \rightarrow \alpha \beta$. We extend these definitions to the case $n=0$ in the natural fashion, so that, if $h \in \operatorname{Hom}_{S}(A, B)$, the homomorphisms $\mu \rightarrow h \mu$ and $\alpha \rightarrow \alpha h$ are the usual maps (denoted above by $h^{*}$ ) corresponding to $h$.

These definitions are independent of the choice of the resolution $X$. Indeed, let $Y$ be another projective resolution of $A$. Let $\beta \in \operatorname{Ext}^{n}(A, B)$, and let $h$ be an element of $\operatorname{Hom}\left(d\left(X_{n}\right), B\right)$ whose image under the iterated connecting homomorphism is $\beta$. Let $g: Y \rightarrow X$ be a map over the identity. We obtain commutative diagrams

$$
\begin{aligned}
& \operatorname{Ext}^{m}\left(d\left(X_{n}\right), C\right) \rightarrow \operatorname{Ext}^{m+n}(A, C) \quad \operatorname{Hom}_{s}\left(d\left(X_{n}\right), B\right) \rightarrow \operatorname{Ext}^{n}(A, B) \\
& \downarrow g^{*} \quad \downarrow \quad \downarrow g^{*} \quad \downarrow \\
& \operatorname{Ext}^{m}\left(d\left(Y_{n}\right), C\right) \rightarrow \operatorname{Ext}^{m+n}(A, C), \quad \operatorname{Hom}_{s}\left(d\left(Y_{n}\right), B\right) \rightarrow \operatorname{Ext}^{n}(A, B),
\end{aligned}
$$

where $g^{*}$ is induced by $g$, and the right-hand vertical maps are the identity. We imbed the first of these in the commutative diagram

$$
\begin{array}{cccc}
\operatorname{Ext}^{m}(B, C) & \stackrel{h^{*}}{\rightarrow} & \operatorname{Ext}^{m}\left(d\left(X_{n}\right), C\right) & \rightarrow \operatorname{Ext}^{m+n}(A, C) \\
\downarrow & \left(g^{*}(h)\right)^{*} \quad g^{*} \downarrow & & \downarrow \\
\operatorname{Ext}^{m}(B, C) & \stackrel{\downarrow}{\longrightarrow} & \operatorname{Ext}^{m}\left(d\left(Y_{n}\right), C\right) & \rightarrow \operatorname{Ext}^{m+n}(A, C)
\end{array}
$$

and from the second we conclude that $g^{*}(h)$ also maps onto $\beta$, so that the rows of (7.6) are the action by $\beta$ defined by using $X$ and $Y$, respectively. Hence the independence of the choice of resolution. A similar proof obtains for the action on Tor.

Let $f \in \operatorname{Hom}_{s}\left(C^{\prime}, C\right)$, where $C^{\prime}$ is also a left $S$-module. Then the diagram

$$
\begin{aligned}
\operatorname{Ext}^{m}\left(B, C^{\prime}\right) & \rightarrow \operatorname{Ext}^{m+n}\left(A, C^{\prime}\right) \\
\downarrow f^{*} & f^{*} \downarrow \\
\operatorname{Ext}^{m}(B, C) & \rightarrow \operatorname{Ext}^{m+n}(A, C)
\end{aligned}
$$

is easily seen to be commutative, where the horizontal maps correspond to an element $\beta \in \operatorname{Ext}^{n}(A, B)$. As a special case, we obtain a commutative diagram

$$
\begin{array}{cc}
\operatorname{Hom}_{s}(B, B) & \rightarrow \operatorname{Ext}^{n}(A, B) \\
\downarrow f^{*} & \downarrow f^{*} \\
\operatorname{Hom}_{s}(B, C) & \rightarrow \operatorname{Ext}^{n}(A, C)
\end{array}
$$

where $f \in \operatorname{Hom}_{S}(B, C)$. Remarking that the image of the identity homomorphism 
in $\operatorname{Hom}_{S}(B, B)$ under the horizontal map is $\beta$, and that its image under $f^{*}$ is $f$, we conclude that $\beta f=f^{*}(\beta)$. Note that by definition a similar statement holds for left operations by elements of degree 0 ; i.e., if $g \in \operatorname{Hom}_{S}(A, B)$ and $\mu \in \operatorname{Ext}^{m}(B, C), g \mu=g^{*}(\mu)$.

Proposition 7.1. Let $X$ be an $S$-projective resolution of the left $S$-module $A$. Let $\beta \in \operatorname{Ext}(A, B)$ be represented by $f \in \operatorname{Hom}\left(X_{n}, B\right)$. Let $Y$ be a projective resolution of $B$, and let

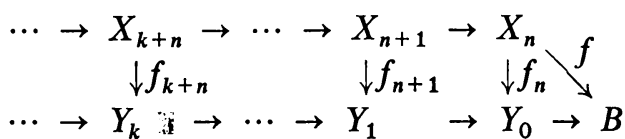

be commutative. Then if $\mu \in \operatorname{Ext}^{m}(B, C)$ is represented by $g \in \operatorname{Hom}_{S}\left(Y_{m}, C\right), \beta \mu$ is represented in $\operatorname{Hom}_{S}\left(X_{m+n}, C\right)$ by $(-1)^{m n} g f_{m+n}$; and if $n \leqq m$, and $\alpha \in \operatorname{Tor}_{m}(D, A)$ is represented by $a \in D \otimes_{S} X_{m}, \quad \alpha \beta$ is represented in $D \otimes_{S} Y_{m-n}$ by $(-1)^{(m+1) n}\left(1 \otimes f_{m}\right)(a)$.

Proof. Consider $\cdots \rightarrow X_{k+1} \rightarrow X_{k} \rightarrow \cdots \rightarrow X_{n}$, suitably renumbered, as a projective resolution of $d\left(X_{n}\right)$. The identity map defines a map of complexes,

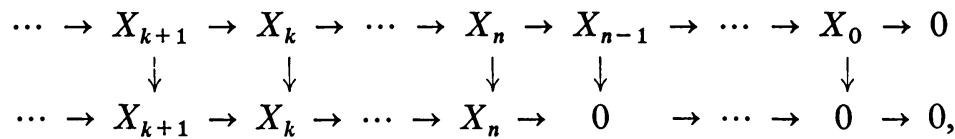

and thus a map: $\operatorname{Ext}^{m}\left(d\left(X_{n}\right), B\right) \rightarrow \operatorname{Ext}^{m+n}(A, B)$. This map differs from the iterated connecting homomorphism by a multiplicative factor of $(-1)^{t}$, where $t=m n+n(n+1) / 2\left[1\right.$, Proposition 7.1, p. 92]. Let $f^{\prime} \in \operatorname{Hom}_{S}\left(d\left(X_{n}\right), B\right)$ be the element represented by $f$; i.e., let $f=f^{\prime} d$, where $d$ is the boundary map on $X$. Then we conclude from the above that $f^{\prime}$ is mapped by the iterated connecting homomorphism onto $(-1)^{t} \beta$, where $t=n(n+1) / 2$. Since $f=f^{\prime} d$, the maps $f_{k}$ of (7.7) define a map over $f^{\prime}$ from a projective resolution of $d\left(X_{n}\right)$ to a projective resolution of $B$. Hence $f^{\prime *}(\mu) \in \operatorname{Ext}^{m}\left(d\left(X_{n}\right), C\right)$ is represented by $g f_{m+n} \in \operatorname{Hom}_{S}\left(X_{m+n}, C\right)$. Therefore the image of $f^{* *}(\mu)$ in $\operatorname{Ext}^{m+n}(A, C)$ under the iterated connecting homomorphism is represented by $(-1)^{t_{1}} g f_{m+n}$, where $t_{1}=m n+n(n+1) / 2$. Hence we conclude that $\beta \mu$ is represented by $(-1)^{t+t_{1}} g f_{m+n}=(-1)^{m n} g f_{m+n}$, which proves the first part of the proposition.

The map: $\operatorname{Tor}_{m}(D, A) \rightarrow \operatorname{Tor}_{m-n}\left(D, d\left(X_{n}\right)\right)$ induced by (7.8) differs from the iterated connecting homomorphism by $(-1)^{s}$, where $s=m n+n(n-1) / 2$. Using this, the second part of the proposition is proved in analogous fashion to the first.

Proposition 7.2. Let $\beta \in \operatorname{Ext}(A, B), \mu \in \operatorname{Ext}(B, C), \alpha \in \operatorname{Ext}(C, E)$, and $\alpha^{\prime} \in \operatorname{Tor}(D, A)$. Then $(\beta \mu) \alpha=\beta(\mu \alpha)$, and $\left(\alpha^{\prime} \beta\right) \mu=\alpha^{\prime}(\beta \mu)$.

The proof is straightforward, using Proposition 7.1. 
It follows from Proposition 7.2 that under the operations defined in this section $\operatorname{Ext}(A, A)$ becomes a graded $\operatorname{ring}$, and $\operatorname{Tor}(D, A)$ becomes a graded $\operatorname{right} \operatorname{Ext}(A, A)-$ module.

8. Relations with other products. For $n \geqq 1$, consider an $S$-exact sequence

$$
0 \rightarrow B \rightarrow E_{n-1} \rightarrow \cdots \rightarrow E_{0} \rightarrow A \rightarrow 0 .
$$

We may associate with such an $n$-fold extension of $B$ over $A$ its characteristic element; i.e., the image in $\operatorname{Ext}_{S}^{n}(A, B)$, under the iterated connecting homomorphism corresponding to (8.1), of the identity homomorphism in $\operatorname{Hom}_{S}(B, B)$. Let $X$ be a projective resolution of $A$. Then we can find maps over the identity of $A$ such that

$$
\begin{aligned}
& 0 \rightarrow d\left(X_{n}\right) \rightarrow X_{n-1} \rightarrow \cdots \rightarrow X_{0} \rightarrow A \rightarrow 0 \\
& h \downarrow \\
& 0 \rightarrow B \longrightarrow E_{n-1} \rightarrow \cdots \rightarrow E_{0} \rightarrow A \rightarrow 0
\end{aligned}
$$

commutes, whence we obtain a commutative diagram

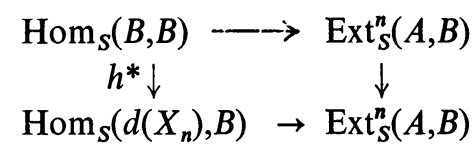

where the horizontal maps are iterated connecting homomorphisms and the right-hand vertical map is the identity. Remarking that the image under $h^{*}$ of the identity homomorphism is $h$, we conclude from the latter that $h$ may be used to define the action on $\operatorname{Ext}_{s}(B, C)$ and $\operatorname{Tor}^{S}(D, A)$ of the characteristic element of (8.1). Then from the commutative diagrams

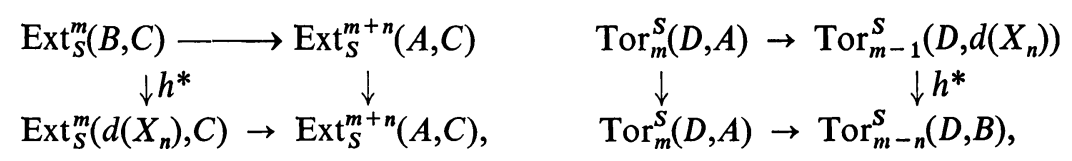

we conclude that these actions coincide with the iterated connecting homomorphisms corresponding to (8.1).

Consider the diagram

$$
\begin{array}{cc}
\operatorname{Hom}_{S}(A, A) & \rightarrow \operatorname{Ext}_{S}^{p}(C, A) \\
\downarrow & \downarrow \\
\operatorname{Ext}_{S}^{n}(A, B) & \rightarrow \operatorname{Ext}_{S}^{n+p}(C, B)
\end{array}
$$

where the vertical maps are iterated connecting homomorphisms corresponding to (8.1), and the horizontal maps are operation by some element $\beta \in \operatorname{Ext}_{S}^{p}(C, A)$. It follows at once, from the same property for similar diagrams in which all maps are iterated connecting homomorphisms, that this diagram commutes or anti-commutes according as $n p$ is even or odd. Remarking that the image in 
$\operatorname{Ext}_{S}(C, A)$ of the identity homomorphism is $\beta$, we conclude that right operation by the image of the identity homomorphism in $\operatorname{Ext}_{S}^{n}(A, B)$ differs from the iterated connecting homomorphism corresponding to (8.1) by a multiplicative factor of $(-1)^{n p}$. But it is easily shown, by induction on $n$, that the image in $\operatorname{Ext}_{S}^{n}(A, B)$ of the identity homomorphism in $\operatorname{Hom}_{S}(A, A)$ differs from the characteristic element of (8.1) by a multiplicative factor of $(-1)^{t}$, where $t=n(n+1) / 2$. (See $[1$, p. 308 , Exercise 1] for the case $n=1$.)

We have proved

Proposition 8.1. The maps from $\operatorname{Ext}_{S}(B, C)$ to $\operatorname{Ext}_{S}(A, C)$ and from $\operatorname{Tor}^{S}(D, A)$ to $\operatorname{Tor}^{S}(D, B)$ corresponding to the characteristic element of (8.1) coincide with the iterated connecting homomorphism corresponding to (8.1). The map from $\operatorname{Ext}_{s}(C, A)$ to $\operatorname{Ext}_{s}^{n+p}(C, B)$ corresponding to the characteristic element of (8.1) differs from the iterated connecting homomorphism corresponding to (8.1) by a multiplicative factor of $(-1)^{s}$, where $s=n p+n(n+1) / 2$.

It is shown in [9] that associating with each $n$-fold extension (8.1) its characteristic element yields a one-to-one correspondence between natural equivalence classes of such $n$-fold extensions and $\operatorname{Ext}_{S}^{n}(A, B)$. In the light of this fact, the first sentence of Proposition 8.1 is seen to characterize the action by elements of $\operatorname{Ext}_{s}^{n}(A, B)$, if $n \geqq 1$.

Given an $m$-fold extension $0 \rightarrow C \rightarrow D_{m-1} \rightarrow \cdots \rightarrow D_{0} \rightarrow B \rightarrow 0$, we may use (8.1) to form an $m+n$-fold extension $0 \rightarrow C \rightarrow D_{m-1} \rightarrow \cdots \rightarrow D_{0} \rightarrow E_{n-1} \rightarrow \cdots \rightarrow E_{0}$ $\rightarrow A \rightarrow 0$. An operation by elements of $\operatorname{Ext}^{n}(A, B)$ mapping $\operatorname{Ext}^{m}(B, C)$ into $\mathrm{Ext}^{m+n}(A, C)$ is thus defined in [9]. It clearly coincides with the iterated connecting homomorphism corresponding to (8.1). Hence, by Proposition 8.1, the product for Ext defined in [9] is the same as that defined in the previous section. $\left(^{3}\right)$

Now let $K$ be a commutative ring, and let $R$ be a $K$-algebra. Let $R^{*}$ be the anti-isomorphic ring to $R$, and let $S=R \otimes_{K} R^{*}$. Then every two-sided $R$-module becomes a left $S$-module, and vice-versa, in the usual fashion. $\left(\left(r_{1} \otimes r_{2}^{*}\right) \cdot m=r_{1} \cdot m \cdot r_{2}\right.$.) If $A$ and $B$ are $S$-modules, $A \otimes_{R} B$ becomes an $S$-module in the natural fashion. $\left(\left(r_{1} \otimes r_{2}^{*}\right)(a \otimes b)=r_{1} a \otimes b r_{2}.\right)$

Assume that $R$ is $K$-projective. Then $S$ is projective both as a left and a right $R$-module. Let $X$ be an $S$-projective resolution of $R$. Since $S$ is $R$-projective, so is $X$. Hence the homology of the complex $X \otimes_{R} X$ is $\operatorname{Tor}^{R}(R, R)$, which is zero in positive degrees. Since $R$ is $K$-projective, $X \otimes_{R} X$ is $S$-projective. Hence $X \otimes_{R} X$ is again an $S$-projective resolution of $R \otimes_{R} R=R$. Hence, if $A$ and $B$ are $S$-modules, the canonical map from $\operatorname{Hom}_{s}(X, A) \otimes_{K} \operatorname{Hom}_{s}(X, B)$ to $\operatorname{Hom}_{S}\left(X \otimes_{R} X, A \otimes_{R} B\right)$ induces a product: $\operatorname{Ext}_{S}^{\mathrm{n}}(R, A) \otimes_{\mathrm{K}} \operatorname{Ext}_{S}^{m}(R, B) \rightarrow$

(3) The correspondence between Ext $^{n}$ and $n$-fold extensions given here differs from that used in [9] by a factor of $(-1)^{t}$, where $t=n(n+1) / 2$. Hence the product of [9] actually differs from those given here by $(-1)^{n m}$. 
$\operatorname{Ext}_{S}^{n+n}\left(R, A \otimes_{R} B\right)$. This is the product $\bigvee$, as given in [1, Exercise 2, p. 229], and used in [5]. Observe that, if $R$ is commutative, $\vee$ can be taken to be defined on $\operatorname{Ext}_{S}(R, A) \otimes_{R} \operatorname{Ext}_{S}(R, B)$.

There is a natural map $(h \rightarrow 1 \otimes h)$ of $\operatorname{Hom}_{S}(X, B)$ into $\operatorname{Hom}_{S}\left(A \otimes_{R} X, A \otimes_{R} B\right)$. The homology of $A \otimes_{R} X$ is $\operatorname{Tor}^{R}(A, R)$, which is zero in positive degrees. Hence $A \otimes_{R} X$ is an acyclic complex over $A \otimes_{R} R=A$, so that there is a uniquely defined map from the homology of $\operatorname{Hom}_{S}\left(A \otimes_{R} X, A \otimes_{R} B\right)$ to $\operatorname{Ext}_{S}\left(A, A \otimes_{R} B\right)$. Combining these two maps, we obtain one from $\operatorname{Ext}_{S}(R, B)$ into $\operatorname{Ext}_{S}\left(A, A \otimes_{R} B\right)$. Combining this in turn with the product defined in the previous section, we obtain a product:

$$
\operatorname{Ext}_{S}(R, A) \times \operatorname{Ext}_{S}(R, B) \rightarrow \operatorname{Ext}_{S}(R, A) \times \operatorname{Ext}_{S}\left(A, A \otimes_{R} B\right) \rightarrow \operatorname{Ext}_{S}\left(R, A \otimes_{R} B\right) .
$$

Proposition 8.2. Let $S, R, A, B$ be as above. The product $\vee$ coincides with the iteration $(8.2)\left({ }^{4}\right)$.

Proof. Referring to the preceding paragraph, let $Y=X \otimes_{R} X$. Define a map $\phi: Y \rightarrow X$ such that, if $x \in X_{p}$ and $y \in X_{q}, \phi(x \otimes y)$ is zero unless $q=0$; and if $q=0, \phi(x \otimes y)=x \varepsilon(y)$, where $\varepsilon: X_{0} \rightarrow R$ is the augmentation map. Then $\phi$ is a map over the identity. Let $\beta \in \operatorname{Ext}_{S}^{n}(R, A)$ be represented by $f \in \operatorname{Hom}_{S}\left(X_{n}, A\right)$. Then $\beta$ is also represented by $f \phi \in \operatorname{Hom}_{s}\left(Y_{n}, A\right)$.

Let $Z$ be an $S$-projective resolution of $A$. Then there is a map $\rho: Z \rightarrow A \otimes_{R} X$ over the identity. If $\mu \in \operatorname{Ext}_{S}^{m}(R, B)$, denote by $\bar{\mu}$ the image of $\mu$ in $\operatorname{Ext}_{S}^{m}\left(A, A \otimes_{R} B\right)$ under the homomorphism used in (8.2). If $\mu$ is represented by $g \in \operatorname{Hom}_{S}\left(X_{m}, B\right)$, $\bar{\mu}$ is represented by $(1 \otimes g) \rho_{m} \in \operatorname{Hom}_{S}\left(Z_{m}, A \otimes_{R} B\right)$. Then if $f_{n}, f_{n+1}, \cdots$ are homomorphisms such that

$$
\begin{array}{llll}
Y_{m+n} \rightarrow \cdots \rightarrow Y_{n+1} & \rightarrow Y_{n} \\
\downarrow f_{m+n} & f_{n+1 \downarrow} \downarrow & f_{n} \downarrow \downarrow \\
Z_{m} \rightarrow \cdots & \rightarrow Z_{1} \rightarrow Z_{0} \rightarrow A
\end{array}
$$

is commutative, $\beta \bar{\mu}$ is represented by $(-1)^{m n}(1 \otimes g) \rho_{m} f_{m+n}$ (Proposition 7.1).

Suppose $f_{n}^{\prime}, f_{n+1}^{\prime}, \cdots$ are homomorphisms such that

$$
\begin{array}{ccc}
Y_{m+n} \rightarrow \cdots \rightarrow \quad Y_{n+1} & \rightarrow Y_{n} \backslash f \phi \\
\downarrow & f_{m+n}^{\prime} \underset{f_{n+1}^{\prime} \downarrow}{f_{n}^{\prime} \downarrow f^{\prime} \downarrow} \\
A \otimes_{R} X_{m} \rightarrow \cdots \rightarrow A \otimes_{R} X_{1} & \rightarrow A \otimes_{R} X_{0} \rightarrow A
\end{array}
$$

is commutative. Then as usual the maps defined by the homomorphisms $\rho_{k-n} f_{k}$ and $f_{k}^{\prime}$ are homotopic; i.e., there are homomorphisms $h_{k}: Y_{k} \rightarrow A \otimes_{R} X_{k-n+1}$ such that, if $k \geqq n$ and $d$ denotes the differentiation on $Y$ and $X, \rho_{k-n} f_{k}-f_{k}^{\prime}=\left(1 \otimes d_{k-n+1}\right) h_{k}$ $+h_{k-1} d_{k}$ (where we take $\left.h_{n-1}=0\right)$. Then $(1 \otimes g) \rho_{m} f_{m+n}-(1 \otimes g) f_{m+n}^{\prime}$

(4) The product $\wedge$ of [1, Exercise 2, p. 229] can be expressed as an iteration: $\operatorname{Tor}^{S}\left(\operatorname{Hom}_{R}(A, B), R\right) \times \operatorname{Ext}_{S}(R, A) \rightarrow \operatorname{Tor}^{S}\left(\operatorname{Hom}_{R}(A, B), A\right) \rightarrow \operatorname{Tor}^{S}(B, R)$. Similar results can be obtained for the products $\bigvee$ and $\Lambda$ of $[1$, pp. 205-206]. 
$=(1 \otimes g)\left(\left(1 \otimes d_{m+1}\right) h_{m+n}+h_{m+n-1} d_{m+n}\right)=\left(1 \otimes g d_{m+1}\right) h_{m+n}+(1 \otimes g) h_{m+n-1} d_{m+n}$. But $g$ is a cocycle, so that $g d_{m+1}=0$. Hence $(1 \otimes g) \rho_{m} f_{m+n}-(1 \otimes g) f_{m+n}^{\prime}$ $=(1 \otimes g) h_{m+n-1} d_{m+n}$, which is a coboundary. Hence $(-1)^{m n}(1 \otimes g) f_{m+n}^{\prime}$ also represents $\beta \bar{\mu}$.

In particular, we can define $f_{p+q}^{\prime}$ on $X_{p} \otimes_{R} X_{q}$ to be 0 unless $p=n$, and to be $(-1)^{n q} f \otimes 1$ in this case. Then (8.3) will be commutative. But then $(1 \otimes g) f_{m+n}^{\prime}$ is $(-1)^{m n} f \otimes g$ on $X_{n} \otimes_{R} X_{m}$ and is 0 on $X_{p} \otimes_{R} X_{q}$ unless $p=n, q=m$. Hence $(-1)^{m n}(1 \otimes g) f_{m+n}^{\prime}$ also represents $\beta \vee \mu$. This proves the proposition.

Note that, in the case $A=B=R$, Proposition 8.2 is the statement that the product $\bigvee$ on $\operatorname{Ext}_{S}(R, R)$ coincides with the product defined in the previous section.

Now let $R$ and $S$ be commutative rings, and let $\varepsilon: S \rightarrow R$ be a ring surjection. Then $\varepsilon$ defines on $R$ the structure of a left $S$-module in the usual fashion. $\left(s \cdot r=\varepsilon(s) r\right.$.) Let $X$ be an $S$-projective resolution of $R$. Then $X \otimes_{S} X$ is still a projective complex over $R \otimes_{S} R=R$, so that there is a map over the identity from $X \otimes_{S} X$ to $X$ which is unique up to homotopy; and a map is thus uniquely defined from the homology of $R \otimes_{S} X \otimes_{S} X$ to $\operatorname{Tor}^{S}(R, R)$. Then the natural map: $\left(R \otimes_{S} X\right) \otimes_{S}\left(R \otimes_{S} X\right) \rightarrow\left(R \otimes_{S} R\right) \otimes_{S} X \otimes_{S} X=R \otimes_{S} X \otimes_{S} X$ defines a product: $\operatorname{Tor}_{n}^{S}(R, R) \otimes_{s} \operatorname{Tor}_{m}^{S}(R, R) \rightarrow \operatorname{Tor}_{n+m}^{S}(R, R)$. This is the product $\pi$ of $[1, \mathrm{p}$. 211]. If $h: X \otimes_{S} X \rightarrow X$ is a map over the identity, and $\phi:\left(R \otimes_{S} X\right) \otimes_{S}\left(R \otimes_{S} X\right)$ $\rightarrow R \otimes_{S} X \otimes_{S} X$ is the map above, $\rightarrow$ is induced on $R \otimes_{S} X$ by $(1 \otimes h) \phi$. Note that $\operatorname{Tor}_{0}^{S}(R, R)=R$ and that on it $\cap$ and ring multiplication coincide.

A graded algebra is skew-symmetric in case, whenever $a$ has degree $n$ and $b$ has degree $m, a b=(-1)^{n m} b a$. $\operatorname{Tor}^{S}(R, R)$ is skew-symmetric under $\pi$; and, if $R$ is commutative, so is $\operatorname{Ext}_{S}(R, R)$ under $\bigvee$. In addition, element of Tor $_{1}$ have square zero. An endomorphism $D$ of degree $k$ of a graded algebra is a derivation in case, whenever $a$ has degree $n, D(a b)=D(a) b+(-1)^{n k} a D(b)$.

Proposition 8.3. Let $\beta \in \operatorname{Ext}_{S}^{1}(R, R)$ with $R$ and $S$ as above. Then right module multiplication by $\beta$ is a derivation of $\operatorname{Tor}^{S}(R, R)$.

Proof. Choose a projective resolution $X$ of $R$, with $X_{0}=S$ and with augmentation $\varepsilon$. Let $Y=X \otimes_{S} X$. Then we can find a map $h: Y \rightarrow X$ over the identity which is the natural isomorphism on $X_{0} \otimes_{S} X$ and $X \otimes_{S} X_{0}$. Let $g \in \operatorname{Hom}_{S}\left(X_{1}, R\right)$ represent $\beta$. Let $f: X \rightarrow X$ be a map with degree -1 such that $\varepsilon f_{1}=g$. Then if $\alpha \in \operatorname{Tor}_{m}^{S}(R, R)$ is represented by $a \in R \otimes_{S} X_{m}, \alpha \beta$ is represented by $(-1)^{m+1}\left(1 \otimes f_{m}\right)(a)$ (Proposition 7.1). Define $f^{\prime}: Y \rightarrow Y$ such that, if $x \in X_{n}$, $y \in X_{m}, f^{\prime}(x \otimes y)=(-1)^{m} f(x) \otimes y+x \otimes f(y)$. Then $f h$ and $h f^{\prime}$ are two maps from $Y$ to $X$ with degree -1 . But by the naturality of $h$ and the $S$-linearity of $f$ they agree on $Y_{1}$. Hence by the usual argument they are homotopic. Hence $(-1)^{n+m+1} f h$ is homotopic to $(-1)^{n+m+1} h f^{\prime}$, which suffices to prove this proposition.

Suppose that 2 has an inverse in $R$. Then $\operatorname{Tor}^{S}(R, R)$ and $\operatorname{Ext}_{S}(R, R)$ contain the images of the exterior $R$-algebras over $\operatorname{Tor}_{1}^{S}(R, R)$ and $\operatorname{Ext}_{S}^{1}(R, R)$, respectively, 
under canonical algebra homomorphisms. From Proposition 8.3 we deduce that, on these images, the pairing between $\operatorname{Tor}^{S}(R, R)$ and $\operatorname{Ext}_{S}(R, R)$ agrees with the usual pairing between the exterior algebra over a module and the exterior algebra over its dual.

9. Contraction and Lie derivation on Tor. Let $K$ be a commutative ring, and let $R$ be a commutative $K$-projective $K$-algebra. Let $S=R \otimes_{K} R$. Referring to the two previous sections, we see that $\operatorname{Ext}_{S}(R, R)$ and $\operatorname{Tor}^{S}(R, R)$ are graded skew-commutative rings (and hence algebras over $\operatorname{Ext}_{S}^{0}(R, R)=R=\operatorname{Tor}_{0}^{S}(R, R)$ ), and that the latter is a graded right module for the former. Let $I$ be the kernel of the canonical epimorphism $\varepsilon: S \rightarrow R$. Then $\operatorname{Ext}_{S}^{1}(R, R)$ is naturally isomorphic, via a connecting homomorphism, to $\operatorname{Hom}_{S}(I, R)$. If $h \in \operatorname{Hom}_{S}(I, R)$, the $K$-endomorphism $\mu$ of $R$ defined by $\mu(x)=h(1 \otimes x-x \otimes 1)$ is a derivation. Conversely, if $\mu$ is a $K$-derivation of $R$, the homomorphism $h: S \rightarrow R$ defined by $h(x \otimes y)=x \mu(y)$ is $S$-linear when restricted to $I$. We have thus an $R$-module isomorphism between $\operatorname{Ext}_{S}^{1}(R, R)$ and the $K$-derivations of $R$. We continue to denote the latter by $T_{R}$. When convenient, we shall regard elements of $T_{R}$ as elements of $\operatorname{Ext}_{S}^{1}(R, R)$ under this isomorphism, and vice-versa.

For $x \in R$, write $d(x)=1 \otimes x \in S$. Then the latter, with its usual left $R$-module structure, is spanned by elements of the form $y d(x)$, with $x, y \in R$. Let $D_{R}$ be $S$ modulo the $R$-module generated by elements of the form $d(x y)-x d(y)-y d(x)$. Then $D_{R}$ is the $R$-module of formal differentials of $R$. $T_{R}$ is isomorphic to $\operatorname{Hom}_{R}\left(D_{R}, R\right)$ via the pairing $(\mu, x d(y)) \rightarrow x \mu(y)$; and $D_{R}$ is isomorphic to $I / I^{2}$ via the map that sends $x d(y)$ onto the coset of $x \otimes y-x y \otimes 1[2]$. $\operatorname{Tor}_{S}^{1}(R, R)$ is naturally isomorphic, by means of a connecting homomorphism, with $R \otimes_{S} I=I / I^{2}$, so that the above pairing gives an isomorphism between $\operatorname{Ext}_{S}^{1}(R, R)$ and $\operatorname{Hom}_{R}\left(\operatorname{Tor}_{1}^{S}(R, R), R\right)$ via the pairing $(\beta, \alpha) \rightarrow \alpha \beta$. More generally we define a homomorphism from $\operatorname{Ext}_{S}^{n}(R, R)$ to $\operatorname{Hom}_{R}\left(\operatorname{Tor}_{n}^{S}(R, R), R\right)$ in a similar fashion. This homomorphism is the same as that used in [5].

We use the dual of this last homomorphism in the following way. For $\mu_{1}, \cdots, \mu_{n} \in T_{R}$, considered as elements of $\operatorname{Ext}_{S}^{1}(R, R)$, and for $\alpha \in \operatorname{Tor}_{n}^{S}(R, R)$,

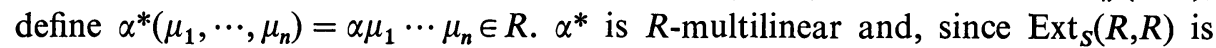
skew-symmetric, is (weakly) alternating in the sense that permuting the arguments changes the value of $\alpha^{*}$ by the sign of the permutation. There is a skewsymmetric "shuffle product" of alternating multilinear maps which is the usual wedge product for forms over a manifold and which is defined as follows: Let $h$ and $k$ be maps of degree $m$ and $n$, respectively, and define

$$
(h \wedge k)\left(\mu_{1}, \cdots, \mu_{m+n}\right)=\Sigma|\sigma| h\left(\mu_{\sigma(1)}, \cdots, \mu_{\sigma(m)}\right) k\left(\mu_{\sigma(m+1)}, \cdots, \mu_{\sigma(m+n)}\right)
$$

where the sum is taken over all permutations $\sigma$ of $1,2, \cdots, m+n$ such that $\sigma(1)<\cdots<\sigma(m)$, and $\sigma(m+1)<\cdots<\sigma(m+n)$, and where $|\sigma|$ is the sign of $\sigma$. Let $\alpha \in \operatorname{Tor}_{m}(R, R)$ and $\beta \in \operatorname{Tor}_{n}(R, R)$. Then, by Proposition 8.3, 


$$
(\alpha \beta) \mu_{1} \cdots \mu_{m+n}=\left(\left(\alpha \mu_{1}\right) \beta+(-1)^{m} \alpha\left(\beta \mu_{1}\right)\right) \mu_{2} \cdots \mu_{m+n} \text {. }
$$

Using this, it is easy to prove by induction that $(\alpha \beta)^{*}=\alpha^{*} \wedge \beta^{*}$ for all $\alpha, \beta \in \operatorname{Tor}^{s}(R, R)$. Hence we have

THEOREM 9.1. Let $R$ be a commutative $K$-projective $K$-algebra, and let $S=R \otimes_{K} R$. Then the map $\alpha \rightarrow \alpha^{*}$ defined above is an $R$-algebra homomorphism from $\operatorname{Tor}^{S}(R, R)$ into the $R$-algebra of the alternating differential forms on $T_{R}$.

Let $\mu \in T_{R}$. Let $\beta_{\mu}$ be the endomorphism of $S$ such that $\beta_{\mu}(x \otimes y)=\mu(x) \otimes y$ $+x \otimes \mu(y)$. Then $\beta_{\mu}$ is a derivation of $S$, and $(1, \mu)$ is a $\beta_{\mu}$-pair on $R$ (cf. $\S 6$ ). Denote the endomorphisms $\operatorname{Tor}^{S, \beta_{\mu}}(\mu, \mu)$ of $\operatorname{Tor}^{S}(R, R)$ and $\operatorname{Ext}_{s, \beta_{\mu}}(\mu, \mu)$ of $\operatorname{Ext}_{s}(R, R)$ by $\theta_{\mu}$.

As in $\S 6$, we easily show

$$
\theta_{\mu} \theta_{\alpha}-\theta_{\alpha} \theta_{\mu}=\theta_{[\mu, \alpha]} .
$$

Proposition 9.2. $\theta_{\mu}$ is a derivation of $\operatorname{Tor}^{S}(R, R)$.

Proof. Let $X$ be an $S$-projective resolution of $R$, and let $(1, \bar{\mu})$ be a $\beta_{\mu}$-pair on $X$ over $(1, \mu)$. Let $Y=X \otimes_{S} X$, and define $\bar{\mu}^{\prime}: Y \rightarrow Y$ such that $\bar{\mu}^{\prime}(x \otimes y)$ $=\bar{\mu}(x) \otimes y+x \otimes \bar{\mu}(y)$. Let $h: Y \rightarrow X$ be a map over the identity. Then $(1, \bar{\mu} h)$ and $\left(1, h \bar{\mu}^{\prime}\right)$ are both $\beta_{\mu}$-pairs over $(1, \mu)$ from $Y$ to $X$ and hence are homotopic. This suffices to prove the proposition.

In the sequel, we will need the following properties of the maps discussed in $\S 6$. Let $(1, \bar{\mu}): D \rightarrow D$ be a $\beta$-pair. To simplify notation, write, e.g., $((f, \mu), \bar{\mu})^{*}$ for $\operatorname{Ext}_{V, \beta}((f, \mu), \bar{\mu})$.

(a) Let $(g, \mu): A \rightarrow B$ be a $\beta$-pair. Let $f \in \operatorname{Hom}_{V}(B, C)$. Then $(f g, f \mu): A \rightarrow C$ is a $\beta$-pair, and

$$
((g, \mu), \bar{\mu}) * \circ \operatorname{Ext}_{V}(f, C)=((f g, f \mu), \bar{\mu})^{*} .
$$

Similarly, if $f \in \operatorname{Hom}_{V}(A, B),(g, \mu): B \rightarrow C$,

$$
\operatorname{Ext}_{V}(f, C) \circ((g, \mu), \bar{\mu})^{*}=((g f, \mu f), \bar{\mu})^{*} .
$$

(b) If in

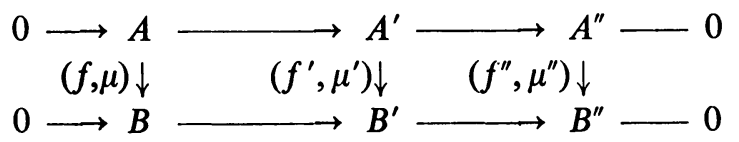

the rows are $V$-exact, the vertical maps are $\beta$-pairs, and the diagram commutes, then

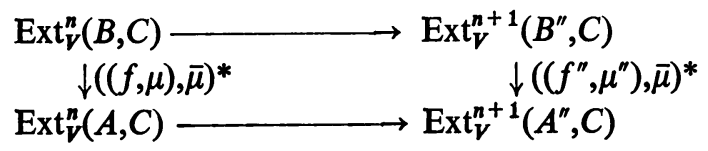

commutes, where the rows are the connecting homomorphisms. 
(c) If $\left(f, \mu^{\prime}\right),(f, \mu): A \rightarrow B$ are $\beta$-pairs, then $\mu^{\prime}-\mu$ is $V$-linear, and

$$
((f, \mu), \bar{\mu})^{*}-\left(\left(f, \mu^{\prime}\right), \bar{\mu}\right)^{*}=\operatorname{Ext}_{V}\left(\mu^{\prime}-\mu, C\right) .
$$

We will also need the analogous statements for Tor. (a) is a special case of the composition law given in [6]. The proof of (b) is analogous to the proof of the similar statement for $V$-linear homomorphisms. (c) is immediate.

Proposition 9.3. Let $\alpha^{\prime} \in \operatorname{Ext}_{s}(R, R)$. Let $\alpha \in \operatorname{Tor}^{S}(R, R)$ or $\operatorname{Ext}_{S}(R, R)$, and $\mu \in T_{R}$. Then $\theta_{\mu}\left(\alpha \alpha^{\prime}\right)=\theta_{\mu}(\alpha) \alpha^{\prime}+\alpha \theta_{\mu}\left(\alpha^{\prime}\right)$.

We prove the proposition for $\alpha^{\prime} \in$ Ext. The proof for Tor is similar. We assume without loss of generality that $\alpha^{\prime} \in \mathrm{Ext}^{n}, \alpha \in \mathrm{Ext}^{m}$.

Let $X$ be an $S$-projective resolution of $R$, and let $(1, \bar{\mu})$ be a $\beta_{\mu}$-pair on $X$ over $(1, \mu)$. Let $f \in \operatorname{Hom}_{s}\left(d\left(X_{n}\right), R\right)$ map onto $\alpha^{\prime}$ under the iterated connecting homomorphism. Write $f^{*}=\operatorname{Ext}_{s}(f, R)$, so that $\alpha \alpha^{\prime}$ is the image under the iterated connecting homomorphism of $f^{*}(\alpha) \in \operatorname{Ext}_{S}\left(d\left(X_{n}\right), R\right)$. Let the restriction of $\bar{\mu}$ to $d\left(X_{n}\right)$ also be denoted by $\bar{\mu}$, so that by (b)

$$
\begin{array}{cc}
\operatorname{Ext}_{S}^{m}\left(d\left(X_{n}\right), R\right) & \rightarrow \operatorname{Ext}_{S}^{m+n}(R, R) \\
\downarrow(\bar{\mu}, \mu)^{*} & \downarrow(\mu, \mu)^{*}=\theta_{\mu} \\
\operatorname{Ext}_{S}^{m}\left(d\left(X_{n}\right), R\right) & \rightarrow \operatorname{Ext}_{S}^{m+n}(R, R)
\end{array}
$$

is commutative. We conclude from this that $\theta_{\mu}\left(\alpha \alpha^{\prime}\right)$ is the image under the iterated connecting homomorphism of $(\vec{\mu}, \mu)^{*}\left(f^{*}(\alpha)\right)$.

Again, we obtain from (b) the commutativity of

$$
\begin{array}{cc}
\operatorname{Hom}_{S}\left(d\left(X_{n}\right), R\right) & \rightarrow \operatorname{Ext}_{S}^{n}(R, R) \\
\downarrow(\bar{\mu}, \mu)^{*} & \downarrow \theta_{\mu} \\
\operatorname{Hom}_{S}\left(d\left(X_{n}\right), R\right) & \rightarrow \operatorname{Ext}_{S}^{n}(R, R)
\end{array}
$$

whence we conclude that $\alpha \theta_{\mu}\left(\alpha^{\prime}\right)$ is the image under the iterated connecting homomorphism of $\left((\bar{\mu}, \mu)^{*}(f)\right)^{*}(\alpha)$. Finally, $\theta_{\mu}(\alpha) \alpha^{\prime}$ is the image of $f^{*}\left((\mu, \mu)^{*}(\alpha)\right)$.

By $(\mathrm{a}), f^{*}\left((\mu, \mu)^{*}(\alpha)\right)=((f, \mu f), \mu)^{*}(\alpha)$, and $(\bar{\mu}, \mu)^{*}\left(f^{*}(\alpha)\right)=((f, f \bar{\mu}), \mu)^{*}$. Hence, by (c), $(\bar{\mu}, \mu)^{*}\left(f^{*}(\alpha)\right)-f^{*}\left((\mu, \mu)^{*}(\alpha)\right)=(\mu f-f \bar{\mu})^{*}(\alpha)=\left((\bar{\mu}, \mu)^{*} f\right)^{*}(\alpha)$. This proves the proposition. We have the immediate

Corollary. $\theta_{\mu}$ is a derivation of $\operatorname{Ext}_{S}(R, R)$.

Let $\mu^{\prime} \in T_{R}$, considered as an element of $\operatorname{Ext}_{S}^{1}(R, R)$. Let $h_{\mu^{\prime}}$ be the corresponding element of $\operatorname{Hom}_{S}(I, R)$. We conclude from (b) and the commutative and exact diagram

$$
\begin{aligned}
0 \rightarrow I & \rightarrow S \rightarrow R \rightarrow 0 \\
& \downarrow \downarrow \\
0 & \downarrow \beta_{\mu} \downarrow \mu \\
& \rightarrow S \rightarrow R \rightarrow 0
\end{aligned}
$$

that 


$$
\begin{array}{cc}
\operatorname{Hom}_{S}(I, R) & \rightarrow \operatorname{Ext}_{S}^{1}(R, R) \\
\downarrow\left(\beta_{\mu}, \mu\right)^{*} & \downarrow \theta_{\mu} \\
\operatorname{Hom}_{S}(I, R) \rightarrow \operatorname{Ext}_{S}^{1}(R, R)
\end{array}
$$

is commutative. Hence $\theta_{\mu}\left(\mu^{\prime}\right)$ corresponds to $\left(\beta_{\mu}, \mu\right)^{*}\left(h_{\mu^{\prime}}\right)=\mu h_{\mu^{\prime}}-h_{\mu^{\prime}} \beta_{\mu^{\prime}}$. $\left(\mu h_{\mu^{\prime}}-h_{\mu^{\prime}} \beta_{\mu}\right)(1 \otimes x-x \otimes 1)=\mu \mu^{\prime}(x)-h_{\mu^{\prime}}(1 \otimes \mu(x)-\mu(x) \otimes 1)=\mu \mu^{\prime}(x)-\mu^{\prime} \mu(x)$ $=\left[\mu, \mu^{\prime}\right](x)$. Hence on $\operatorname{Ext}^{1}(R, R)$

$$
\theta_{\mu}\left(\mu^{\prime}\right)=\left[\mu, \mu^{\prime}\right] .
$$

Remark also that on $\operatorname{Ext}^{0}(R, R)=R=\operatorname{Tor}_{0}(R, R), \theta_{\mu}=\mu$.

Combining Proposition 9.3 with the subsequent remarks, we conclude that, for $\alpha \in \operatorname{Tor}_{n}(R, R)$

$$
\theta_{\mu}(\alpha)^{*}\left(\mu_{1}, \cdots, \mu_{n}\right)=\mu\left(\alpha^{*}\left(\mu_{1}, \cdots, \mu_{n}\right)\right)-\sum_{i=1}^{n} \alpha^{*}\left(\mu_{1}, \cdots,\left[\mu, \mu_{i}\right], \cdots, \mu_{n}\right) .
$$

Hence $\theta_{\mu}$ is analogous to the Lie derivation on differential forms.

For $\mu \in T_{R}$, considered as an element of $\operatorname{Ext}^{1}(R, R)$, define an endomorphism $c_{\mu}$ of $\operatorname{Tor}(R, R)$ by

$$
c_{\mu}(\alpha)=\alpha \mu
$$

Then $c_{\mu}$ is a derivation, by Proposition 8.3. It is immediate that, if $\alpha \in \operatorname{Tor}_{n}^{S}(R, R)$,

$$
c_{\mu}(\alpha)^{*}\left(\mu_{1}, \cdots, \mu_{n-1}\right)=\alpha^{*}\left(\mu, \mu_{1}, \cdots, \mu_{n-1}\right)
$$

so that $c_{\mu}$ is analogous to the usual contraction operator on differential forms.

As a special case of Proposition 9.3 we obtain the familiar relationship

$$
\theta_{\mu} c_{\mu^{\prime}}-c_{\mu^{\prime}} \theta_{\mu}=c_{\left[\mu, \mu^{\prime}\right]}
$$

10. Formal differentiation on $\operatorname{Tor}^{S}(R, R)$. We shall be concerned in this section with the existence of an endomorphism $d$ of $\operatorname{Tor}^{S}(R, R)$, of degree 1 , which plays the role of the differentiation of differential forms. One of the properties which such an endomorphism should possess is the usual one that

$$
c_{\mu} d+d c_{\mu}=\theta_{\mu}
$$

If $d$ satisfies (10.1) and if $\alpha \in \operatorname{Tor}_{n}^{S}(R, R)$,

$d(\alpha)^{*}\left(\mu_{1}, \cdots, \mu_{n+1}\right)=\left(c_{\mu_{1}}(d(\alpha))\right)^{*}\left(\mu_{2}, \cdots, \mu_{n+1}\right)=\left(\theta_{\mu_{1}}(\alpha)-d\left(c_{\mu_{1}}(\alpha)\right)\right)^{*}\left(\mu_{2}, \cdots, \mu_{n+1}\right)$.

Using this and (9.1), it is easy to prove by induction on $n$

PROPOSITION 10.1. If $d$ is an endomorphism of $\operatorname{Tor}^{S}(R, R)$ of degree 1 , satisfying (10.1), and if $D$ denotes the usual differentiation operator for differential forms (Equation (4.3)), then $d(\alpha)^{*}=D\left(\alpha^{*}\right)$ for all $\alpha \in \operatorname{Tor}^{S}(R, R)$. 
In what follows, we shall define such a $d$ on $\operatorname{Tor}^{S}(R, R)$, by defining it on a particular complex whose homology is $\operatorname{Tor}^{S}(R, R)$. It will be an extension of the canonical derivation of $R$ into the formal differentials, will have square zero and will be a derivation of $\operatorname{Tor}^{S}(R, R)$. Because we do not have a functorial definition, the verification that $d$ possesses the requisite properties involves a good deal of explicit computation, and the extent to which these properties determine $d$ remains undetermined.

We shall proceed under the assumption that $R$ is $K$-projective. This could be avoided by replacing Tor ${ }^{S}$ and $\operatorname{Ext}_{S}$ by the relative functors $\operatorname{Tor}^{(S, K)}$ and $\operatorname{Ext}_{(S, K)}$ throughout $\S \S 7-9$, since the resolution of $R$ that we will use in the sequel is an $(S, K)$-projective resolution which is $S$-projective whenever $R$ is $K$-projective. This replacement would require only notational changes, and the remark that the definitions and results of [6] apply equally well to the relative functors.

Let $X_{n}$ be the tensor product over $K$ of $n+2$ copies of $R$. Let $X_{n}$ have the $S$-module structure such that

$$
(x \otimes y) \cdot\left(x_{0} \otimes \cdots \otimes x_{n+1}\right)=x x_{0} \otimes x_{1} \otimes \cdots \otimes x_{n} \otimes x_{n+1} y .
$$

For $n \geqq 1$, define a boundary operator $\Delta: X_{n} \rightarrow X_{n-1}$ such that

$$
\Delta\left(x_{0} \otimes \cdots \otimes x_{n+1}\right)=\sum_{i=0}^{n}(-1)^{i} x_{0} \otimes \cdots \otimes x_{i} x_{i+1} \otimes \cdots \otimes x_{n+1} .
$$

Define the augmentation from $X_{0}=S$ to $R$ to be $\varepsilon$, the canonical epimorphism. Then $X$ is an $S$-complex over $R$. It has a homotopy sending $x_{0} \otimes \cdots \otimes x_{n+1}$ onto $1 \otimes x_{0} \otimes \cdots \otimes x_{n+1}$. Since $R$ is $K$-projective, each $X_{n}$ can be written as the tensor product of $S$ with a $K$-projective module. Hence $X$ is an $S$-projective resolution of $R$. This is the standard resolution of [1, p. 174].

There is a map over the identity from $X \otimes_{S} X$ to $X$ such that

$$
\begin{aligned}
\left(x_{0} \otimes \cdots \otimes x_{n+1}\right) \otimes\left(y_{0} \otimes \cdots \otimes y_{m+1}\right) \\
\quad \rightarrow \Sigma \pm x_{0} y_{0} \otimes z_{1} \otimes \cdots \otimes z_{n+m} \otimes x_{n+1} y_{m+1}
\end{aligned}
$$

where the sum is taken over all permutations $z_{1}, \cdots, z_{n+m}$ of $x_{1}, \cdots x_{n}, y_{1}, \cdots, y_{m}$ such that $x_{i}$ precedes $x$, whenever $i<j$ and similarly for the $y$ 's, and where the sign of each term is taken as that of the corresponding permutation. This map makes $X$ into an associative and skew-symmetric algebra, on which the boundary is a derivation [1, pp. 218-219].

For $\mu \in T_{R}$, let $\bar{c}_{\mu}$ be the endomorphism of degree -1 of $X$ such that

$$
\bar{c}_{\mu}\left(x_{0} \otimes \cdots \otimes x_{n+1}\right)=x_{0} \mu\left(x_{1}\right) \otimes x_{2} \otimes \cdots \oplus x_{n+1} .
$$

Then $\bar{c}_{\mu}$ anti-commutes with the boundary. Further, if $h_{\mu} \in \operatorname{Hom}_{S}(I, R)$ is the element corresponding to $\mu$, i.e., if $h_{\mu}$ is the restriction to $I$ of the homomorphism 
from $S$ to $R$ sending $x \otimes y$ onto $x \mu(y)$, then $\varepsilon \bar{c}_{\mu}=-h_{\mu} \Delta$ on $X_{1}$. Hence, by Proposition 7.1, the endomorphism $1 \otimes \bar{c}_{\mu}$ of $R \otimes_{S} X$ induces $c_{\mu}$ on $\operatorname{Tor}^{S}(R, R)$.

Again, for $\mu \in T_{R}$, let $\bar{\mu}$ be the endomorphism of degree 0 of $X$ such that

$$
\bar{\mu}\left(x_{0} \otimes \cdots \otimes x_{n+1}\right)=\sum_{i=0}^{n+1} x_{0} \otimes \cdots \otimes \mu\left(x_{i}\right) \otimes \cdots \otimes x_{n+1} .
$$

Then $(1, \bar{\mu})$ is a $\beta_{\mu}$-pair over $(1, \mu)$.

Let $Y_{n}$ be the tensor product over $K$ of $n+1$ copies of $R$, with the $R$-module structure such that

$$
x \cdot\left(x_{0} \otimes \cdots \otimes x_{n}\right)=x x_{0} \otimes x_{1} \otimes \cdots \otimes x_{n} .
$$

We define auxiliary endomorphisms $\alpha, p, f_{\mu, i}$ and $\Delta_{k}$ of degree $1,0,0$, and -1 , respectively, such that, on $Y_{n}$,

$$
\begin{array}{ll}
\alpha\left(x_{0} \otimes \cdots \otimes x_{n}\right) & =1 \otimes x_{0} \otimes \cdots \otimes x_{n}, \\
p\left(x_{0} \otimes \cdots \otimes x_{n}\right) & =(-1)^{n} x_{n} \otimes x_{0} \otimes \cdots \otimes x_{n-1}, \\
f_{\mu, i}\left(x_{0} \otimes \cdots \otimes x_{n}\right) & =x_{0} \otimes \cdots \otimes \mu\left(x_{i}\right) \otimes \cdots \otimes x_{n}, \\
\Delta_{k}\left(x_{0} \otimes \cdots \otimes x_{n}\right) & =(-1)^{k} x_{0} \otimes \cdots \otimes x_{k} x_{k+1} \otimes \cdots \otimes x_{n} \quad(k<n), \\
\Delta_{n}\left(x_{0} \otimes \cdots \otimes x_{n}\right) & =(-1)^{n} x_{0} x_{n} \otimes x_{1} \otimes \cdots \otimes x_{n-1},
\end{array}
$$

where $\mu \in T_{R}$. Remark that $p^{n+1}=1$.

The homomorphism from $R \otimes_{S} X_{n}$ to $Y_{n}$ such that

$$
x \otimes\left(x_{0} \otimes \cdots \otimes x_{n+1}\right) \rightarrow x x_{0} x_{n+1} \otimes x_{1} \otimes \cdots \otimes x_{n}
$$

is an $R$-module isomorphism. The boundary map induced on $Y_{n}$ is given by

$$
\Delta=\sum_{j=0}^{n} \Delta_{j}
$$

The homology of $Y$ under this boundary is $\operatorname{Tor}^{S}(R, R)$. The product induced on $Y$ by (10.2) is such that

$$
\left(x_{0} \otimes \cdots \otimes x_{n}\right) \otimes\left(y_{0} \otimes \cdots \otimes y_{m}\right) \rightarrow \Sigma \pm x_{0} y_{0} \otimes z_{1} \otimes \cdots \otimes z_{n+m}
$$

where the $z$ 's and the sign are determined as before. $Y$ thus becomes a skewsymmetric $R$-algebra, with a product that induces $\pi$ on $\operatorname{Tor}^{S}(R, R)$.

If we also denote by $\theta_{\mu}$ the endomorphism of $Y$ obtained from (10.3) and inducing $\theta_{\mu}$ on $\operatorname{Tor}^{S}(R, R)$, it is easy to verify that on $Y_{n}$

$$
\theta_{\mu}=\sum_{j=0}^{n} f_{\mu, j}
$$

Again, if we also denote by $c_{\mu}$ the endomorphism of $Y$ corresponding to $1 \otimes \bar{c}_{\mu}$, it is clear that 


$$
c_{\mu}\left(x_{0} \otimes \cdots \otimes x_{n}\right)=x_{0} \mu\left(x_{1}\right) \otimes x_{2} \otimes \cdots \otimes x_{n} .
$$

It is easily verified that, already on $Y, \theta_{\mu}$ and $c_{\mu}$ are derivations, and

$$
\theta_{\mu} c_{\mu^{\prime}}-c_{\mu^{\prime}} \theta_{\mu}=c_{\left[\mu, \mu^{\prime}\right]} .
$$

Now define an endomorphism $\bar{d}$ of $Y$ of degree 1 such that on $Y_{n}$

On $Y_{n}$,

$$
\tilde{d}=\sum_{j=0}^{n} \alpha p^{j}
$$

$$
\begin{array}{ll}
\Delta_{k} p=p \Delta_{k-1} \quad(k>0), & \Delta_{k} \alpha=-\alpha \Delta_{k-1} \quad(0<k \leqq n), \\
\Delta_{0} p=\Delta_{n}, & \Delta_{0} \alpha=1, \\
\Delta_{n+1} \alpha & =-p .
\end{array}
$$

Hence

$$
\begin{aligned}
\Delta p & =p \Delta+(1-p) \Delta_{n}, \\
\Delta \alpha+\alpha \Delta & =1-p+\alpha \Delta_{n} .
\end{aligned}
$$

From (10.4) and (10.5) it follows that

$$
\Delta p^{k}=\sum_{j=0}^{k-1}(1-p) p^{k-1} \Delta_{n-j}+p^{k} \Delta \quad(0 \leqq k \leqq n) .
$$

Using (10.6) and (10.7), a long but straightforward computation yields

$$
\Delta \bar{d}+\bar{d} \Delta=0 \text {. }
$$

Hence $d$ induces a map on $\operatorname{Tor}^{S}(R, R)$. We will show first that the latter satisfies (10.1). To this end, we define

$$
f_{\mu}=\sum_{i=1}^{n} \sum_{j=0}^{n-i} \alpha p^{j} f_{\mu, i}
$$

We remark that on $Y_{n}$, if $i \geqq 1$,

$$
f_{\mu, i} \Delta_{j}= \begin{cases}\Delta_{j} f_{\mu, i} & (i<j), \\ \Delta_{i}\left(f_{\mu, i}+f_{\mu, i+1}\right) & (i=j), \\ \Delta_{j} f_{\mu, i+1} & (i>j) .\end{cases}
$$

In what follows, much of the computation required to pass from one step to the next is long, but all is straightforward. Using (10.8), we find that on $Y_{n}$

$$
\sum_{i=1}^{n-k-1} f_{\mu, i} \Delta=\Delta \sum_{i=1}^{n-k} f_{\mu, i}-\sum_{j=n-k}^{n} \Delta_{j} f_{\mu, n-k}-\Delta_{0} f_{\mu, 1}
$$

and, using (10.7), that 


$$
\Delta \sum_{i=1}^{n} \sum_{k=0}^{n-i} p^{k} f_{\mu, i}=\sum_{k=0}^{n-1} p^{k} \Delta \sum_{i=1}^{n-k} f_{\mu, i}+\sum_{i=1}^{n} \sum_{j=0}^{n-i-1}\left(p^{j}-p^{n-i}\right) \Delta_{n-j} f_{\mu, i}
$$

Combining this with (10.9) and using the fact that $\Delta_{0} f_{\mu, 1}=c_{\mu}$, we obtain

$$
\begin{aligned}
\Delta \sum_{i=1}^{n} \sum_{k=0}^{n-i} p^{k} f_{\mu, i}= & \sum_{i=1}^{n-1} \sum_{k=0}^{n-1-i} p^{k} f_{\mu, i} \Delta \\
& +\sum_{i=1}^{n} \sum_{k=i}^{n} p^{n-k} \Delta_{k} f_{\mu, i}+\sum_{k=0}^{n-1} p^{k} c_{\mu} .
\end{aligned}
$$

Now, using (10.6),

$$
\Delta f_{\mu}=\sum_{i=1}^{n}\left(1-p^{n-i+1}\right) f_{\mu, i}+\alpha\left(\Delta_{n}-\Delta\right) \sum_{i=1}^{n} \sum_{j=0}^{n-i} p f_{\mu, i}
$$

and from (10.4)

$$
\Delta_{n} \sum_{i=1}^{n} \sum_{j=0}^{n-i} p^{j} f_{\mu, i}=\sum_{i=1}^{n} \sum_{k=i}^{n} p^{n-k} \Delta_{k} f_{\mu, i}
$$

Finally, using that $c_{\mu} \alpha=f_{\mu, 0}$ and $f_{\mu, 0} p^{j}=p^{j} f_{\mu, n-j+1}$ for $1 \leqq j \leqq n+1$, we obtain

$$
c_{\mu} \bar{d}=\sum_{i=0}^{n} p^{n-i+1} f_{\mu, i} .
$$

Combining (10.10) through (10.13) yields

$$
c_{\mu} d+\Delta f_{\mu}=\theta_{\mu}-f_{\mu} \Delta-\bar{d} c_{\mu} .
$$

Hence the map induced by $\bar{d}$ satisfies (10.1).

Next we will show that $\bar{d}$ induces a derivation on $\operatorname{Tor}^{S}(R, R)$. To this end we define a bilinear function on $X$ by defining $h: X_{n} \times X_{m} \rightarrow X_{n+m+2} . h$ is defined so that

$$
h\left(\left(x_{0} \otimes \cdots \otimes x_{n}\right),\left(y_{0} \otimes \cdots \otimes y_{m}\right)\right)=\Sigma \pm 1 \otimes z_{1} \otimes \cdots \otimes z_{n+m+2}
$$

where the sum ranges over all permutations $z_{1}, \cdots, z_{n+m+2}$ of

satisfying

$$
x_{0}, y_{0}, x_{1}, \cdots, x_{n}, y_{1}, \cdots, y_{m}
$$

The order of the $x$ 's is a cyclic permutation of $x_{0}, \cdots, x_{n}$, and similarly for the $y$ 's; and $x_{0}$ precedes $y_{0}$,

and where the sign of each term of the sum is the sign of the corresponding permutation.

For brevity, write $a=x_{0} \otimes \cdots \otimes x_{n}, b=y_{0} \otimes \cdots \otimes y_{m}$. Then, if $1 \leqq k \leqq n+m+1, \Delta_{k} h(a, b)$ is a sum of terms of the form

$$
\pm(-1)^{k} 1 \otimes z_{1} \otimes \cdots \otimes z_{k} z_{k+1} \otimes \cdots \otimes z_{n+m+2} .
$$


If $z_{k}=x_{i}$ and $z_{k+1}=y_{j}$, then, unless $i=j=0, z_{1}, \cdots, z_{k-1}, z_{k+1}, z_{k}, z_{k+2}$, $\cdots, z_{n+m+2}$ also satisfies (10.14), and $\Delta_{k}$ of this term cancels (10.15). The same argument applies if $z_{k}=y_{i}$ and $z_{k+1}=x_{j}$.

The terms of (10.15) for which $z_{k}=x_{i}$ and $z_{k+1}=x_{i+1}$ (or $z_{k+1}=x_{0}$ if $i=n$ ) are precisely the terms of $h(\Delta(a), b)$. The similar terms for $y$ 's comprise $(-1)^{n} h(a, \Delta(b))$. Those for which $z_{k}=x_{0}$ and $z_{k+1}=y_{0}$ are the terms of $-d(a b)$. Hence

$$
\sum_{k=1}^{n+m+1} \Delta_{k} h(a, b)-h(\Delta(a), b)-(-1)^{n} h(a, \Delta(b))=-\vec{d}(a, b) .
$$

If $\pm 1 \otimes z_{1} \otimes \cdots \otimes z_{n+m+2}$ is a term of $h(a, b)$, and if $z_{n+m+2} \neq y_{0}$, then $\pm 1 \otimes z_{n+m+2} \otimes z_{1} \otimes \cdots \otimes z_{n+m+1}$ is also a term of $h(a, b)$, and

$\Delta_{n+m+2}\left( \pm 1 \otimes z_{1} \otimes \cdots \otimes z_{n+m+2}\right)+\Delta_{0}\left( \pm 1 \otimes z_{n+m+2} \otimes z_{1} \otimes \cdots \otimes z_{n+m+1}\right)=0$.

Similarly, if $z_{1} \neq x_{0}$, then $\pm 1 \otimes z_{2} \otimes \cdots \otimes z_{n+m+2} \otimes z_{1}$ is also a term of $h(a, b)$, and

$$
\Delta_{0}\left( \pm 1 \otimes z_{1} \otimes \cdots \otimes z_{n+m+2}\right)+\Delta_{n+m+2}\left( \pm 1 \otimes z_{2} \otimes \cdots \otimes z_{n+m+2} \otimes z_{1}\right)=0 .
$$

Hence

$\left(\Delta_{0}+\Delta_{n+m+2}\right) h(a, b)=\Sigma \pm x_{0} \otimes z_{2} \otimes \cdots \otimes z_{n+m+2}-\Sigma \pm y_{0} \otimes z_{1} \otimes \cdots \otimes z_{n+m+1}$ where the sums run over all terms such that $x_{0}, z_{2}, \cdots, z_{n+m+2}$ (respectively $\left.z_{1}, \cdots, z_{n+m+1}, y_{0}\right)$ are permutations satisfying (10.14), and where the sign is the sign of the permutation $x_{0}, y_{0}, x_{1}, \cdots, x_{n}, y_{1}, \cdots, y_{m} \rightarrow x_{0}, z_{2}, \cdots, z_{n+m+2}$ (respectively $\left.\rightarrow y_{0}, z_{1}, \cdots, z_{n+m+1}\right)$. Hence

$$
\left(\Delta_{0}+\Delta_{n+m+2}\right) h(a, b)=(-1)^{n} a d(b)+d(a) b .
$$

(10.16) and (10.17) yield

$$
\bar{d}(a) b+(-1)^{n} a d(b)-\bar{d}(a b)=\Delta h(a, b)-h(\Delta(a), b)-(-1)^{n} h(a, \Delta(b)) .
$$

Hence $d$ induces a derivation on $\operatorname{Tor}^{S}(R, R)$.

It is easy to verify that, if $x \in R$, the image of the formal differential $d(x)$ in $\operatorname{Tor}_{1}^{S}(R, R)$, under the isomorphism defined in $\S 9$, is represented by $d(x)=1 \otimes x \in Y_{1}$. Hence, on $\operatorname{Tor}_{0}^{S}(R, R)=R, d$ induces the homomorphism corresponding to formal differentiation.

Define

$$
d=(1-p) d \text {. }
$$

Then, using (10.6) and the fact that, on $Y_{n}, \Delta_{n+1} \alpha=-p$, we obtain

$$
d-d=\Delta \alpha d-\alpha d \Delta .
$$

Hence $d$ and $d$ induce the same endomorphism of $\operatorname{Tor}(R, R)$. Since $\sum_{i=0}^{n+1} p^{i}(1-p)=0$ on $Y_{n+1}, d^{2}=0$. We have shown 
THEOREM 10.2. There is an endomorphism of degree 1 and square zero on the complex $Y$ such that the endomorphism thereby induced on $\operatorname{Tor}^{S}(R, R)$ is a derivation which extends the formal differentiation from $R$ to $\operatorname{Tor}_{1}^{S}(R, R)$, and which satisfies equation (10.1) above.

There is on $E\left(D_{R}\right)$ a uniquely defined derivation $d$ of degree 1 and square zero, which extends the formal differentiation from $R$ to $D_{R}$. Indeed, by the usual properties of an exterior algebra, we have only to remark that such a $d$ can be defined uniquely on $D_{R}$; the definition being such that $d(x d(y))=d(x) d(y)$. There is also, for each $\mu \in T_{R}$, a uniquely defined derivation $\theta_{\mu}$ of degree 0 such that, on $R, \theta_{\mu}=\mu$ and, on $D_{R}, \theta_{\mu}(x d(y))=\mu(x) d(y)+x d(\mu(y))$, and a uniquely defined derivation $c_{\mu}$ of degree -1 such that, on $D_{R}, c_{\mu}(x d(y))=x \mu(y)$.

The isomorphism from $D_{R}$ onto $\operatorname{Tor}_{1}^{S}(R, R)$ extends canonically to an algebra homomorphism $h: E\left(D_{R}\right) \rightarrow \operatorname{Tor}^{s}(R, R)$. We have remarked above that $h d=d h$ on $R$. Since $d$ has square zero and is a derivation on $\operatorname{Tor}^{S}(R, R)$ we must also have $h d=d h$ on $D_{R}$. Hence, since $d$ is a derivation on $\operatorname{Tor}(R, R)$, we have the commutativity relation on all of $E\left(D_{R}\right)$. Similarly, $h \theta_{\mu}=\theta_{\mu} h$ and $h c_{\mu}=c_{\mu} h$, since the endomorphisms are all derivations and agree on degrees 0 and 1 .

\section{BIBLIOGRA PHY}

1. H. Cartan and S. Eilenberg, Homological algebra, Princeton Univ. Press, Princeton, N. J., 1956.

2. P. Cartier, Dérivations dans les corps, École Norm. Sup., Paris, 1956, Exp. 13.

3. J. Herz, Pseudo-algèbres de Lie, C. R. Acad. Sci. Paris 236 (1953), 1935-1937.

4. G. Hochschild, Relative homological algebra, Trans. Amer. Math. Soc. 82 (1956), 246-269.

5. G. Hochschild, B. Kostant and A. Rosenberg, Differential forms on regular affine algebras, Trans. Amer. Math. Soc. 102 (1962), 383-408.

6. G. Hochschild and A. Rosenberg, Operations on Tor and Ext, Proc. Amer. Math. Soc. 13 (1962), 88-92.

7. I. Kaplansky, Projective modules, Ann. of Math. (2) 68 (1958), 372-377.

8. R. Palais, The cohomology of Lie rings, pp. 130-137, Proc. Sympos. Pure Math., Vol. III, Amer. Math. Soc., Providence, R. I., 1961.

9. N. Yoneda, On the homology theory of modules, J. Fac. Sci. Univ. Tokyo Sect. I 8 (1954), 193-227.

Columbia University, New YorK, New York 